\title{
Comprehensive Study and Experimental Validation of U-shaped Probe Extrinsic Fiber Optic Sensor for the Measurement of Refractive Index at Various Temperatures using a Tunable Light Source
}

\author{
Dr. S. Venkateswara Rao ${ }^{1} \&$ S. Srinivasulu ${ }^{2 *}$
}

Corresponding Author: S. Srinivasulu* (siluverusrinivasulu@gmail.com)

${ }^{1}$ Professor of Physics, Department of Physics, College of Engineering Hyderabad, J N T University Hyderabad, Telangana State, India.

${ }^{2}$ UGC-Senior Research Fellow, Department of Physics, College of Engineering Hyderabad, J N T University Hyderabad, Telangana State, India.

\begin{abstract}
Extensive detection performances due to sensitivity to external and internal perturbations in the fiber structure makes the optical fiber sensors highly superior over the conventional sensors. The fiber optic sensors with clad removed fibers at some portion along its length play a vital role in the determination of refractive index of various liquids at several wavelengths and at several temperatures. In the present paper a refractive index sensor has been developed to investigate the performance and experimental validation in the measurement of R.I. values of liquids at various temperatures using a tunable light source capable of emitting light at the wavelengths of $630 \mathrm{~nm}, 660 \mathrm{~nm}, 820 \mathrm{~nm}$ and $850 \mathrm{~nm}$. The $\mathrm{U}$-shaped glass probe connected between the tunable light source and an optical detector using two multimode PCS fibers of $200 / 230 \mu \mathrm{m}$, acts as a clad removed portion of the sensor which is called as sensing zone or sensing region. In the working principle of the sensor, the $U$-shaped glass rod immersed into each mixture prepared with the combination of Toluene and Acetonitrile, the light launched from the source reaching the detector was noted at various temperatures and by tuning the wavelength of the source to $630 \mathrm{~nm}, 660 \mathrm{~nm}, 820 \mathrm{~nm}$ and $850 \mathrm{~nm}$. From the data obtained, the sensor was calibrated to measure the refractive index of various liquids at different temperatures and wavelengths. Exploiting all the advantages offered by the fiber optic communication systems, the sensor was expected to be rugged, robust, reliable, and durable offering the sensitivity in the range of the order of $10^{-5}$.
\end{abstract}

Keywords: Acetonitrile, PCS fibers, Refractive index, Sensitivity, Toluene, Tunable light source, U-shaped glass probe, Various temperatures

\section{INTRODUCTION}

The refractive index studies have been used widely in numerous analytical applications to identify chemical substances that include food safety, environmental monitoring and medical examination [1-5]. Because of its advantages of fast response time, multiplexing capability, remote sensing, application as OTDR, high bandwidth, immune to EMI, light in size and cheap in cost, the fiber based refractive index sensors have been explored extensively across the world. Based on the parameter to be measure, optical fiber sensors with different geometrical designs have been developed, which includes fiber Bragg grating sensors, multimode fiber interferometer and long period fiber grating, and so on [68]. However, these sensors require complex manufacturing processes, expensive equipments and hence high production cost. Because of its superior performance, optical coupling, machinability, robustness and handling of plastic optical fibers (POF) have received tremendous attention in the recent past [9-13]. For the improvement in refractive index sensitivity of POFs, various kinds of structural modifications have been employed in tapering mode fibers, making hole in fibers and side polishing of fibers, etc. sensors [14-21]. Plastic optical fibers are more suitable for refractive index sensors based on the intensity modulation schemes with multimode characteristics [22-24]. The shape, the operating wavelength and modifications in surface should be combined as a multiple solutions to improve the sensitivity of the sensors. It was recognized intuitively to detect the change in the light intensity due to the variation in refractive index in the visible light operation [25-26]. The U-shaped plastic optical fiber sensor provides the possibility of dip-type probe allowing the light collector and source placed on one side. The scheme of side polishing and bending of the fiber increases the evanescent wave power converting the lower order modes 
into higher order modes reducing the number of modes [27].

In the present work a comprehensive study and experimental validation of a U-shaped probe PCS fiber optic sensor operating between $10^{\circ} \mathrm{C}$ to $60^{\circ} \mathrm{C}$ has been under taken, employing a tunable light source capable of producing the radiation at $630 \mathrm{~nm}, 660 \mathrm{~nm}, 820 \mathrm{~nm}$ and $850 \mathrm{~nm}$.

\section{EXPERIMENTAL DETAILS}

The experimentation has been carried out using four different wavelengths of the source and employing an experimental setup consisting of a U-shaped glass rod connected between the source and a light detector, operating between $10^{\circ} \mathrm{C}$ to $60^{\circ} \mathrm{C}$. A set of binary mixtures with a combination of Toluene and Acetonitrile have been chosen to prepare mixtures with different ratios making the total volume equivalent to $20 \mathrm{ml}$.

Thus, the chemical mixtures exhibit a range of refractive indices ranging between $1.37121 \mathrm{n}_{\mathrm{D}}$ to $1.50915 \mathrm{n}_{\mathrm{D}}$, at the wavelength of $5893 \AA$ (sodium vapour lamp), with the help of which the sensor can be calibrated to determine the refractive index of various liquids either dark or bright within afore side range of values. In the preparation of mixtures Acetonitrile is added systematically to the Toluene with increased mole fraction values in total mixture of Toluene and Acetonitrile. Thus, the mole fractions of Acetonitrile values have been calculated using the standard mathematical expression, which plays a significant role in the variation of refractive index of whole mixture.

Table.1: Standard properties of Toluene and Acetonitrile.

\begin{tabular}{|c|c|c|}
\hline Properties & Toluene $\left(\mathbf{C}_{7} \mathbf{H}_{8}\right)$ \\
\hline
\end{tabular}

The refractive indices of all the mixtures have been determined at different temperatures ranging from $10^{\circ} \mathrm{C}$ to $60^{\circ} \mathrm{C}$ in steps of $5^{\circ} \mathrm{C}$ using automatic digital refractometer of model number RX-7000i (Atago make, Japan). The corresponding refractive index data along with mole fractions of Acetonitrile have been recorded in the tabular form [table-2].

Table.2: Mole fraction of Acetonitrile in Toluene + Acetonitrile chemical mixtures and Refractive indices of mixtures at various temperatures (from $10^{\circ} \mathrm{C}$ to $60^{\circ} \mathrm{C}$ ).

\begin{tabular}{|c|c|c|c|c|c|c|c|c|c|c|c|c|}
\hline \multirow{2}{*}{$\begin{array}{c}\text { S. } \\
\text { No } \\
\cdot\end{array}$} & $\begin{array}{c}\text { Mole } \\
\text { fraction } \\
\text { of } \\
\begin{array}{c}\text { Acetonitri } \\
\text { le }\end{array}\end{array}$ & \multicolumn{10}{|c|}{ Refractive Index at various temperatures } \\
\cline { 3 - 13 } & $\mathbf{1 0}^{\circ} \mathrm{C}$ & $\mathbf{1 5}^{\circ} \mathrm{C}$ & $\mathbf{2 0}^{\circ} \mathrm{C}$ & $\mathbf{2 5}^{\circ} \mathrm{C}$ & $\mathbf{3 0}{ }^{\circ} \mathrm{C}$ & $\mathbf{3 5}^{\circ} \mathrm{C}$ & $\mathbf{4 0}^{\circ} \mathrm{C}$ & $\mathbf{4 5}^{\circ} \mathrm{C}$ & $\mathbf{5 0}^{\circ} \mathrm{C}$ & $\mathbf{5 5}^{\circ} \mathrm{C}$ & $\mathbf{6 0}^{\circ} \mathrm{C}$ \\
\hline 1 & 0.00000 & $\begin{array}{c}1.5091 \\
5\end{array}$ & $\begin{array}{c}1.5059 \\
1\end{array}$ & $\begin{array}{c}1.5017 \\
1\end{array}$ & $\begin{array}{c}1.4977 \\
0\end{array}$ & $\begin{array}{c}1.4932 \\
5\end{array}$ & $\begin{array}{c}1.4897 \\
4\end{array}$ & $\begin{array}{c}1.4858 \\
2\end{array}$ & $\begin{array}{c}1.4829 \\
3\end{array}$ & $\begin{array}{c}1.4779 \\
5\end{array}$ & $\begin{array}{c}1.4750 \\
9\end{array}$ & $\begin{array}{c}1.4710 \\
2\end{array}$ \\
\hline
\end{tabular}




\begin{tabular}{|c|c|c|c|c|c|c|c|c|c|c|c|c|}
\hline 2 & 0.18321 & $\begin{array}{c}1.4821 \\
1\end{array}$ & $\begin{array}{c}1.4779 \\
5\end{array}$ & $\begin{array}{c}1.4745 \\
2\end{array}$ & $\begin{array}{c}1.4708 \\
6\end{array}$ & $\begin{array}{c}1.4667 \\
5\end{array}$ & $\begin{array}{c}1.4620 \\
5\end{array}$ & $\begin{array}{c}1.4587 \\
2\end{array}$ & $\begin{array}{c}1.4549 \\
5\end{array}$ & $\begin{array}{c}1.4508 \\
3\end{array}$ & $\begin{array}{c}1.4463 \\
7\end{array}$ & $\begin{array}{c}1.4428 \\
3\end{array}$ \\
\hline 3 & 0.33541 & $\begin{array}{c}1.4600 \\
9\end{array}$ & $\begin{array}{c}1.4559 \\
7\end{array}$ & $\begin{array}{c}1.4520 \\
8\end{array}$ & $\begin{array}{c}1.4479 \\
5\end{array}$ & $\begin{array}{c}1.4440 \\
3\end{array}$ & $\begin{array}{c}1.4401 \\
9\end{array}$ & $\begin{array}{c}1.4362 \\
3\end{array}$ & $\begin{array}{c}1.4328 \\
7\end{array}$ & $\begin{array}{c}1.4280 \\
4\end{array}$ & $\begin{array}{c}1.4241 \\
3\end{array}$ & $\begin{array}{c}1.4208 \\
7\end{array}$ \\
\hline 4 & 0.46386 & $\begin{array}{c}1.4419 \\
7\end{array}$ & $\begin{array}{c}1.4382 \\
3\end{array}$ & $\begin{array}{c}1.4342 \\
6\end{array}$ & $\begin{array}{c}1.4300 \\
7\end{array}$ & $\begin{array}{c}1.4268 \\
2\end{array}$ & $\begin{array}{c}1.4218 \\
7\end{array}$ & $\begin{array}{c}1.4180 \\
9\end{array}$ & $\begin{array}{c}1.4137 \\
6\end{array}$ & $\begin{array}{c}1.4100 \\
7\end{array}$ & $\begin{array}{c}1.4059 \\
6\end{array}$ & $\begin{array}{c}1.4024 \\
3\end{array}$ \\
\hline 5 & 0.57372 & $\begin{array}{c}1.4259 \\
6 \\
\end{array}$ & $\begin{array}{c}1.4218 \\
7 \\
\end{array}$ & $\begin{array}{c}1.4177 \\
2 \\
\end{array}$ & $\begin{array}{c}1.4142 \\
8 \\
\end{array}$ & $\begin{array}{c}1.4104 \\
9 \\
\end{array}$ & $\begin{array}{c}1.4059 \\
6 \\
\end{array}$ & $\begin{array}{c}1.4020 \\
4\end{array}$ & $\begin{array}{c}1.3980 \\
4 \\
\end{array}$ & $\begin{array}{c}1.3935 \\
6 \\
\end{array}$ & $\begin{array}{c}1.3900 \\
5 \\
\end{array}$ & $\begin{array}{c}1.3860 \\
4\end{array}$ \\
\hline 6 & 0.66874 & $\begin{array}{c}1.4107 \\
5\end{array}$ & $\begin{array}{c}1.4071 \\
4\end{array}$ & $\begin{array}{c}1.4030 \\
8\end{array}$ & $\begin{array}{c}1.3991 \\
4\end{array}$ & $\begin{array}{c}1.3950 \\
7\end{array}$ & $\begin{array}{c}1.3910 \\
4\end{array}$ & $\begin{array}{c}1.3870 \\
5\end{array}$ & $\begin{array}{c}1.3832 \\
3\end{array}$ & $\begin{array}{c}1.3790 \\
4\end{array}$ & $\begin{array}{c}1.3754 \\
3\end{array}$ & $\begin{array}{c}1.3712 \\
3\end{array}$ \\
\hline 7 & 0.75175 & $\begin{array}{c}1.3991 \\
4\end{array}$ & $\begin{array}{c}1.3953 \\
5\end{array}$ & $\begin{array}{c}1.3915 \\
3\end{array}$ & $\begin{array}{c}1.3875 \\
2\end{array}$ & $\begin{array}{c}1.3832 \\
3\end{array}$ & $\begin{array}{c}1.3798 \\
5\end{array}$ & $\begin{array}{c}1.3748 \\
8\end{array}$ & $\begin{array}{c}1.3708 \\
2\end{array}$ & $\begin{array}{c}1.3671 \\
3\end{array}$ & $\begin{array}{c}1.3630 \\
8\end{array}$ & $\begin{array}{c}1.3590 \\
8\end{array}$ \\
\hline 8 & 0.82488 & $\begin{array}{c}1.3860 \\
4\end{array}$ & $\begin{array}{c}1.3825 \\
8\end{array}$ & $\begin{array}{c}1.3781 \\
4\end{array}$ & $\begin{array}{c}1.3743 \\
9\end{array}$ & $\begin{array}{c}1.3701 \\
8\end{array}$ & $\begin{array}{c}1.3668 \\
4\end{array}$ & $\begin{array}{c}1.3628 \\
5\end{array}$ & $\begin{array}{c}1.3587 \\
5\end{array}$ & $\begin{array}{c}1.3549 \\
2\end{array}$ & $\begin{array}{c}1.3509 \\
8\end{array}$ & $\begin{array}{c}1.3469 \\
6\end{array}$ \\
\hline 9 & 0.88981 & $\begin{array}{c}1.3771 \\
2\end{array}$ & $\begin{array}{c}1.3727 \\
9\end{array}$ & $\begin{array}{c}1.3685 \\
7\end{array}$ & $\begin{array}{c}1.3647 \\
8\end{array}$ & $\begin{array}{c}1.3610 \\
6\end{array}$ & $\begin{array}{c}1.3570 \\
6\end{array}$ & $\begin{array}{c}1.3526 \\
8\end{array}$ & $\begin{array}{c}1.3488 \\
6\end{array}$ & $\begin{array}{c}1.3448 \\
9\end{array}$ & $\begin{array}{c}1.3408 \\
6\end{array}$ & $\begin{array}{c}1.3366 \\
5\end{array}$ \\
\hline 10 & 0.94783 & $\begin{array}{c}1.3664 \\
7 \\
\end{array}$ & $\begin{array}{c}1.3620 \\
2\end{array}$ & $\begin{array}{c}1.3580 \\
4\end{array}$ & $\begin{array}{c}1.3540 \\
2 \\
\end{array}$ & $\begin{array}{c}1.3509 \\
8 \\
\end{array}$ & $\begin{array}{c}1.3464 \\
3 \\
\end{array}$ & $\begin{array}{c}1.3420 \\
7 \\
\end{array}$ & $\begin{array}{c}1.3381 \\
2 \\
\end{array}$ & $\begin{array}{c}1.3340 \\
7 \\
\end{array}$ & $\begin{array}{c}1.3300 \\
8\end{array}$ & $\begin{array}{c}1.3260 \\
4\end{array}$ \\
\hline 11 & 1.00000 & $\begin{array}{c}1.3570 \\
6\end{array}$ & $\begin{array}{c}1.3530 \\
4\end{array}$ & $\begin{array}{c}1.3488 \\
6\end{array}$ & $\begin{array}{c}1.3451 \\
7\end{array}$ & $\begin{array}{c}1.3410 \\
8\end{array}$ & $\begin{array}{c}1.3370 \\
5\end{array}$ & $\begin{array}{c}1.3330 \\
6\end{array}$ & $\begin{array}{c}1.3290 \\
3\end{array}$ & $\begin{array}{c}1.3251 \\
1\end{array}$ & $\begin{array}{c}1.3210 \\
5\end{array}$ & $\begin{array}{c}1.3172 \\
1\end{array}$ \\
\hline
\end{tabular}

Unlike density of the substance, the refractive index of the material can be decided by the polarizing nature of the substance. All the four contributions of the polarization put together influence the loss of energy in the medium and hence the refractive index of the medium. More is the polarization of the medium, more is the dielectric constant

of the medium, and hence the refractive index of the medium. Therefore, the relation between refractive index and dielectric constant is given by $\boldsymbol{\varepsilon}_{\mathbf{r}}=\mathbf{n}^{\mathbf{2}}$ and by using this equation, dielectric constant of all the mixtures at different temperatures have been calculated and tabulated [table-3].

Table.3: Mole fraction of Acetonitrile in Toluene + Acetonitrile chemical mixtures and Dielectric constant of mixtures at various temperatures (from $10^{\circ} \mathrm{C}$ to $60^{\circ} \mathrm{C}$ ).

\begin{tabular}{|c|c|c|c|c|c|c|c|c|c|c|c|c|}
\hline \multirow{2}{*}{$\begin{array}{c}\text { S. } \\
\text { No. }\end{array}$} & \multirow{2}{*}{$\begin{array}{c}\text { Mole } \\
\text { fraction of } \\
\text { Acetonitrile }\end{array}$} & \multicolumn{11}{|c|}{ Dielectric constant at various temperatures } \\
\hline & & $10^{\circ} \mathrm{C}$ & $5^{\circ} \mathrm{C}$ & $0^{\circ} \mathrm{C}$ & $25^{\circ} \mathrm{C}$ & $30^{\circ} \mathrm{C}$ & $35^{\circ} \mathrm{C}$ & $40^{\circ} \mathrm{C}$ & $45^{\circ} \mathrm{C}$ & $50^{\circ} \mathrm{C}$ & $\mathbf{5 5}^{\circ} \mathrm{C}$ & $60^{\circ} \mathrm{C}$ \\
\hline 1 & 0.00000 & 2.27753 & 26776 & 25513 & 2.24311 & 2.22980 & 2.21934 & 2.20766 & .19908 & 2.18434 & 2.17589 & 2.16390 \\
\hline 2 & 0.18 & 19665 & 2.18434 & 2.17421 & 2.16343 & 2.15136 & 2.13759 & 2.12786 & 2.11688 & 2.10491 & 2.09198 & 2.08176 \\
\hline 3 & 0.33541 & 13186 & 2.11985 & 2.10853 & 2.09656 & 2.08522 & 2.07415 & 2.06276 & 2.05312 & 2.03930 & 2.02815 & 2.01887 \\
\hline 4 & 0.46386 & 8 & 1 & 0 & 0 & 2 & 2.02171 & 2 & 2 & 0 & 7672 & 96681 \\
\hline 5 & 0.57372 & 2.03336 & 2.02171 & 2.00993 & 2.00019 & 1.98948 & 1.97672 & 1.96572 & 1.95452 & 1.94201 & 1.93224 & 1.92111 \\
\hline 6 & 0.66874 & 99022 & 1.98004 & 1.96863 & 1.95759 & 1.94622 & 1.93500 & 1.92391 & 1.91332 & 1.90175 & 1.89181 & 1.88027 \\
\hline 7 & 0 . & צנוני & 1.94701 & 1.93636 & 1.92 & 1.91332 & 1.9039 & 1.89030 & 1.87915 & 1.86904 & 1.85798 & 1.84710 \\
\hline 8 & 0.82488 & 1.92111 & 1.91153 & 1.89927 & 1.88895 & 1.87739 & 1.86825 & 1.85736 & 1.84620 & 1.83581 & 1.82515 & 1.81430 \\
\hline 9 & 0.88981 & 1.89646 & 1.88455 & 1.87298 & 1.86262 & 1.85248 & 1.84161 & 1.82974 & 1.81942 & 1.80873 & 1.79791 & 1.78663 \\
\hline 10 & 0.94783 & 36724 & 1.85510 & 1.84427 & 1.83337 & 1.82515 & 1.81287 & 1.80115 & 1.79056 & 1.77974 & 1.76911 & 1.75838 \\
\hline 11 & 1.00000 & .84161 & 1.83072 & 1.81942 & 1.80948 & 1.79850 & 1.78770 & 1.77705 & 1.76632 & 1.75592 & 1.74517 & 1.73504 \\
\hline
\end{tabular}

If the fiber connected between the source and detector is perfect in all aspects, a negligible amount of loss may be observed. Any deviation or perturbation in the fiber medium leads to a substantial loss of light signal transmitting from source to receiver end. A PCS fiber with clad removed portion at some region along its length 
offers a substantial loss of light from the fiber. Based on this principle a uniform U-shaped borosilicate glass rod has been used as a sensing region by connecting the first end of the rod to a tunable light source and other end is connected to the light detector using multimode PCS fibers. With a view to enhance the sensitivity of the sensor, the glass rod employed was taken in the shape of a "U'.
Immersing the U-shaped glass rod into the first mixture and simultaneously heating with a temperature bath, the light reaching the receiver was noted down; maintaining a fixed depth of immersion of glass rod into the mixture as $3 \mathrm{~cm}$. By sequentially taking the mixtures one by one, the above procedure was repeated for all the mixtures and data was collected and tabulated.

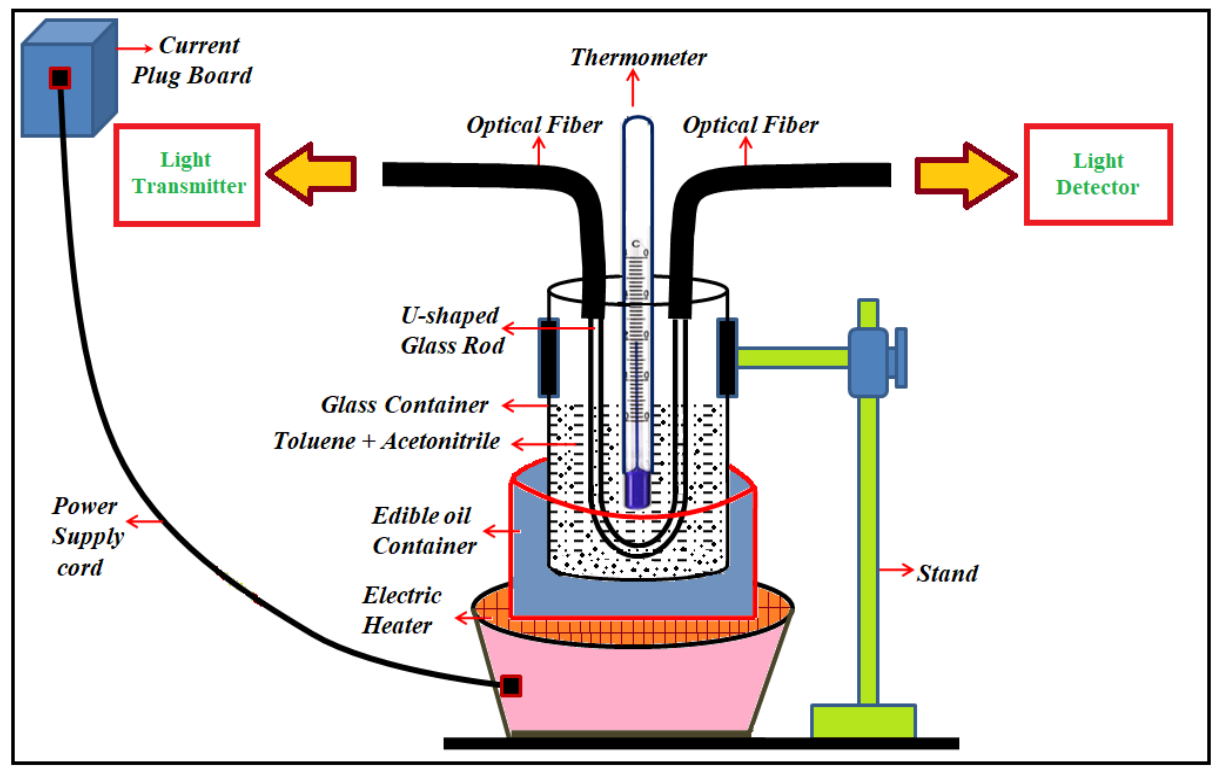

Fig.1: Experimental arrangement of U-shaped Probe Extrinsic Fiber Optic Refractive Index Sensor.

Table.4: Mole fraction of Acetonitrile in Toluene + Acetonitrile chemical mixtures and Output power at various temperatures (from $10^{\circ} \mathrm{C}$ to $60^{\circ} \mathrm{C}$ ), when operating wavelength of the source $630 \mathrm{~nm}$.

Output Power when air surrounding the U-shaped glass rod $-24.80 \mathrm{dBm}$ (at $30^{\circ} \mathrm{C}$ )

\begin{tabular}{|c|c|c|c|c|c|c|c|c|c|c|c|c|}
\hline \multirow{2}{*}{$\begin{array}{c}\text { S. } \\
\text { No. }\end{array}$} & \multirow{2}{*}{$\begin{array}{c}\text { Mole } \\
\text { fraction of } \\
\text { Acetonitrile } \\
\end{array}$} & \multicolumn{11}{|c|}{ Output Power (dBm) at various temperatures } \\
\hline & & $1^{\circ} \mathrm{C}$ & $15^{\circ} \mathrm{C}$ & $20^{\circ} \mathrm{C}$ & $25^{\circ} \mathrm{C}$ & $3^{\circ} \mathrm{C}$ & $35^{\circ} \mathrm{C}$ & $40^{\circ} \mathrm{C}$ & $45^{\circ} \mathrm{C}$ & $\mathbf{5 0}^{\circ} \mathrm{C}$ & $\mathbf{5 5}^{\circ} \mathrm{C}$ & $60^{\circ} \mathrm{C}$ \\
\hline 1 & 0.00000 & $\begin{array}{c}- \\
44.90 \\
\end{array}$ & 44.53 & $\begin{array}{c}- \\
44.13 \\
\end{array}$ & $\begin{array}{c}- \\
43.73 \\
\end{array}$ & $\begin{array}{c}- \\
43.30 \\
\end{array}$ & 42.93 & 42.53 & 42.23 & 41.87 & 41.47 & 41.07 \\
\hline 2 & 0.18321 & $\begin{array}{c}- \\
42.37 \\
\end{array}$ & $\begin{array}{c}- \\
41.97 \\
\end{array}$ & $\begin{array}{c}- \\
41.60 \\
\end{array}$ & $\begin{array}{c}- \\
41.27 \\
\end{array}$ & $\begin{array}{c}- \\
40.90 \\
\end{array}$ & $\begin{array}{c}- \\
40.53 \\
\end{array}$ & $\begin{array}{c}- \\
40.27 \\
\end{array}$ & $\begin{array}{c}- \\
39.97 \\
\end{array}$ & $\begin{array}{c}- \\
39.53 \\
\end{array}$ & $\begin{array}{c}- \\
39.10\end{array}$ & $\begin{array}{c}- \\
38.70 \\
\end{array}$ \\
\hline 3 & 0.33541 & $\begin{array}{c}- \\
40.40 \\
\end{array}$ & $\begin{array}{c}- \\
40.03 \\
\end{array}$ & $\begin{array}{c}- \\
39.67 \\
\end{array}$ & $\begin{array}{c}- \\
39.30 \\
\end{array}$ & $\begin{array}{c}- \\
38.93 \\
\end{array}$ & $\begin{array}{c}- \\
38.57 \\
\end{array}$ & $\begin{array}{c}- \\
38.17 \\
\end{array}$ & $\begin{array}{c}- \\
37.83 \\
\end{array}$ & 37.33 & $\begin{array}{c}- \\
36.83 \\
\end{array}$ & $\begin{array}{c}- \\
36.43 \\
\end{array}$ \\
\hline 4 & 0.46386 & $\begin{array}{c}- \\
38.73 \\
\end{array}$ & $\begin{array}{c}- \\
38.37 \\
\end{array}$ & $\begin{array}{c}- \\
37.97 \\
\end{array}$ & $\begin{array}{c}- \\
37.57 \\
\end{array}$ & $\begin{array}{c}- \\
37.20 \\
\end{array}$ & $\begin{array}{c}- \\
36.63 \\
\end{array}$ & $\begin{array}{c}- \\
36.20 \\
\end{array}$ & $\begin{array}{c}- \\
35.77 \\
\end{array}$ & $\begin{array}{c}- \\
35.50 \\
\end{array}$ & $\begin{array}{c}- \\
35.07 \\
\end{array}$ & $\begin{array}{c}- \\
34.63 \\
\end{array}$ \\
\hline 5 & 0.57372 & $\begin{array}{c}- \\
36.87 \\
\end{array}$ & $\begin{array}{c}- \\
36.53 \\
\end{array}$ & $\begin{array}{c}- \\
36.17 \\
\end{array}$ & $\begin{array}{c}- \\
35.83 \\
\end{array}$ & $\begin{array}{c}- \\
35.57 \\
\end{array}$ & $\begin{array}{c}- \\
35.07 \\
\end{array}$ & $\begin{array}{c}- \\
34.60 \\
\end{array}$ & $\begin{array}{c}- \\
34.37 \\
\end{array}$ & $\begin{array}{c}- \\
34.07 \\
\end{array}$ & $\begin{array}{c}- \\
33.73 \\
\end{array}$ & $\begin{array}{c}- \\
33.37 \\
\end{array}$ \\
\hline 6 & 0.66874 & $\begin{array}{c}- \\
35.57 \\
\end{array}$ & $\begin{array}{c}- \\
35.27 \\
\end{array}$ & $\begin{array}{c}- \\
34.77 \\
\end{array}$ & $\begin{array}{c}- \\
34.47 \\
\end{array}$ & 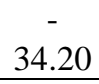 & $\begin{array}{c}- \\
33.83 \\
\end{array}$ & $\begin{array}{c}- \\
33.47 \\
\end{array}$ & 33.13 & 32.80 & 32.53 & $\begin{array}{c}- \\
32.20 \\
\end{array}$ \\
\hline 7 & 0.75175 & $\begin{array}{c}- \\
34.60 \\
\end{array}$ & $\begin{array}{c}- \\
34.34 \\
\end{array}$ & $\begin{array}{c}- \\
33.90 \\
\end{array}$ & $\begin{array}{c}- \\
33.53 \\
\end{array}$ & $\begin{array}{c}- \\
33.17 \\
\end{array}$ & $\begin{array}{c}- \\
32.87 \\
\end{array}$ & $\begin{array}{c}- \\
32.50 \\
\end{array}$ & $\begin{array}{c}- \\
32.17 \\
\end{array}$ & $\begin{array}{c}- \\
31.83 \\
\end{array}$ & $\begin{array}{c}- \\
31.50\end{array}$ & $\begin{array}{c}- \\
31.13 \\
\end{array}$ \\
\hline 8 & 0.82488 & $\begin{array}{c}- \\
33.37 \\
\end{array}$ & $\begin{array}{c}- \\
33.07 \\
\end{array}$ & $\begin{array}{c}- \\
32.73 \\
\end{array}$ & $\begin{array}{c}- \\
32.47 \\
\end{array}$ & $\begin{array}{c}- \\
32.10 \\
\end{array}$ & $\begin{array}{c}- \\
31.80 \\
\end{array}$ & $\begin{array}{c}- \\
31.47 \\
\end{array}$ & 31.10 & 30.77 & 30.37 & 29.97 \\
\hline
\end{tabular}




\begin{tabular}{|c|c|c|c|c|c|c|c|c|c|c|c|c|}
\hline 9 & 0.88981 & $\begin{array}{c}- \\
32.67\end{array}$ & $\begin{array}{c}- \\
32.30 \\
\end{array}$ & $\begin{array}{c}- \\
31.97 \\
\end{array}$ & $\begin{array}{c}- \\
31.67\end{array}$ & $\begin{array}{c}- \\
31.27 \\
\end{array}$ & 30.93 & $\begin{array}{c}- \\
30.60 \\
\end{array}$ & $\begin{array}{c}- \\
30.27 \\
\end{array}$ & $\begin{array}{c}- \\
29.87 \\
\end{array}$ & 29.37 & $\begin{array}{c}- \\
28.77 \\
\end{array}$ \\
\hline 10 & 0.94783 & $\begin{array}{c}- \\
31.77\end{array}$ & $\begin{array}{c}- \\
31.37\end{array}$ & $\begin{array}{c}- \\
31.07\end{array}$ & $\begin{array}{c}- \\
30.70\end{array}$ & $\begin{array}{c}- \\
30.47\end{array}$ & 30.03 & $\begin{array}{c}- \\
29.60\end{array}$ & $\begin{array}{c}- \\
29.07\end{array}$ & $\begin{array}{c}- \\
28.60\end{array}$ & $\begin{array}{c}- \\
28.10\end{array}$ & $\begin{array}{c}- \\
27.53\end{array}$ \\
\hline 11 & 1.00000 & $\begin{array}{c}- \\
30.77\end{array}$ & $\begin{array}{c}- \\
30.63\end{array}$ & $\begin{array}{c}- \\
30.27\end{array}$ & $\begin{array}{c}- \\
29.90\end{array}$ & $\begin{array}{c}- \\
29.50\end{array}$ & $\begin{array}{c}- \\
28.90\end{array}$ & $\begin{array}{c}- \\
28.37\end{array}$ & $\begin{array}{c}- \\
27.87\end{array}$ & $\begin{array}{c}- \\
27.33\end{array}$ & $\begin{array}{c}- \\
26.90\end{array}$ & $\begin{array}{c}- \\
26.33\end{array}$ \\
\hline
\end{tabular}

The experimentation was further carried out by tuning the wavelength of the source to $660 \mathrm{~nm}, 820 \mathrm{~nm} \& 850 \mathrm{~nm}$ and corresponding output power data was recorded [table 57].

Table.5: Mole fraction of Acetonitrile in Toluene + Acetonitrile chemical mixtures and Output power at various temperatures (from $10^{\circ} \mathrm{C}$ to $60^{\circ} \mathrm{C}$ ), when operating wavelength of the source $660 \mathrm{~nm}$.

\begin{tabular}{|c|c|c|c|c|c|c|c|c|c|c|c|c|}
\hline \multirow{2}{*}{$\begin{array}{c}\text { S. } \\
\text { No. }\end{array}$} & \multirow{2}{*}{$\begin{array}{c}\text { Mole } \\
\text { fraction of } \\
\text { Acetonitrile } \\
\end{array}$} & \multicolumn{11}{|c|}{ Output Power (dBm) at various temperatures } \\
\hline & & $\mathbf{1 0}^{\circ} \mathrm{C}$ & $15^{\circ} \mathrm{C}$ & $20^{\circ} \mathrm{C}$ & $25^{\circ} \mathrm{C}$ & $30^{\circ} \mathrm{C}$ & $35^{\circ} \mathrm{C}$ & $40^{\circ} \mathrm{C}$ & $45^{\circ} \mathrm{C}$ & $\mathbf{5 0}^{\circ} \mathrm{C}$ & $55^{\circ} \mathrm{C}$ & $60^{\circ} \mathrm{C}$ \\
\hline 1 & 0.00000 & $\begin{array}{c}- \\
45.23 \\
\end{array}$ & $\begin{array}{c}- \\
44.87 \\
\end{array}$ & 44.47 & 44.07 & $\begin{array}{c}- \\
43.63 \\
\end{array}$ & 43.27 & $\begin{array}{c}- \\
42.87 \\
\end{array}$ & 42.57 & 42.03 & 41.80 & 41.40 \\
\hline 2 & 0.18321 & $\begin{array}{c}- \\
42.50 \\
\end{array}$ & $\begin{array}{c}- \\
42.03 \\
\end{array}$ & $\begin{array}{c}- \\
41.73 \\
\end{array}$ & $\begin{array}{c}- \\
41.47 \\
\end{array}$ & $\begin{array}{c}- \\
41.23 \\
\end{array}$ & $\begin{array}{c}- \\
40.87 \\
\end{array}$ & $\begin{array}{c}- \\
40.60 \\
\end{array}$ & $\begin{array}{c}- \\
40.27 \\
\end{array}$ & $\begin{array}{c}- \\
39.77 \\
\end{array}$ & $\begin{array}{c}- \\
39.43 \\
\end{array}$ & $\begin{array}{c}- \\
39.03 \\
\end{array}$ \\
\hline 3 & 0.33541 & $\begin{array}{c}- \\
40.73 \\
\end{array}$ & $\begin{array}{c}- \\
40.37 \\
\end{array}$ & $\begin{array}{c}- \\
40.00 \\
\end{array}$ & $\begin{array}{c}- \\
39.63 \\
\end{array}$ & $\begin{array}{c}- \\
39.27\end{array}$ & $\begin{array}{c}- \\
38.90 \\
\end{array}$ & $\begin{array}{c}- \\
38.50\end{array}$ & $\begin{array}{c}- \\
38.17\end{array}$ & $\begin{array}{c}- \\
37.67 \\
\end{array}$ & $\begin{array}{c}- \\
37.17 \\
\end{array}$ & $\begin{array}{c}- \\
36.77 \\
\end{array}$ \\
\hline 4 & 0.46386 & $\begin{array}{c}- \\
39.07 \\
\end{array}$ & $\begin{array}{c}- \\
38.70 \\
\end{array}$ & 38.30 & 37.87 & $\begin{array}{c}- \\
37.53 \\
\end{array}$ & $\begin{array}{c}- \\
36.97 \\
\end{array}$ & $\begin{array}{c}- \\
36.53 \\
\end{array}$ & 36.10 & 35.83 & 35.40 & 34.97 \\
\hline 5 & 0.57372 & $\begin{array}{c}- \\
37.20\end{array}$ & $\begin{array}{c}- \\
36.70 \\
\end{array}$ & $\begin{array}{c}- \\
36.30 \\
\end{array}$ & $\begin{array}{c}- \\
36.10\end{array}$ & $\begin{array}{c}- \\
35.87\end{array}$ & $\begin{array}{c}- \\
35.40\end{array}$ & $\begin{array}{c}- \\
34.83 \\
\end{array}$ & -34.7 & $\begin{array}{c}- \\
34.40\end{array}$ & $\begin{array}{c}- \\
34.07\end{array}$ & $\begin{array}{c}- \\
33.70\end{array}$ \\
\hline 6 & 0.66874 & $\begin{array}{c}- \\
35.90 \\
\end{array}$ & $\begin{array}{c}- \\
35.60 \\
\end{array}$ & 35.10 & $\begin{array}{c}- \\
34.77 \\
\end{array}$ & $\begin{array}{c}- \\
34.53 \\
\end{array}$ & $\begin{array}{c}- \\
34.17 \\
\end{array}$ & $\begin{array}{c}- \\
33.80 \\
\end{array}$ & $\begin{array}{c}- \\
33.47 \\
\end{array}$ & $\begin{array}{c}- \\
33.13 \\
\end{array}$ & $\begin{array}{c}- \\
32.87 \\
\end{array}$ & $\begin{array}{c}- \\
32.53 \\
\end{array}$ \\
\hline 7 & 0.75175 & 34.77 & $\begin{array}{c}- \\
34.57 \\
\end{array}$ & $\begin{array}{c}- \\
34.23 \\
\end{array}$ & $\begin{array}{c}- \\
33.87 \\
\end{array}$ & $\begin{array}{c}- \\
33.47 \\
\end{array}$ & $\begin{array}{c}- \\
33.20 \\
\end{array}$ & $\begin{array}{c}- \\
32.83 \\
\end{array}$ & $\begin{array}{c}- \\
32.50 \\
\end{array}$ & $\begin{array}{c}- \\
32.17 \\
\end{array}$ & $\begin{array}{c}- \\
31.83 \\
\end{array}$ & $\begin{array}{c}- \\
31.47 \\
\end{array}$ \\
\hline 8 & 0.82488 & ${ }^{-}-\overline{73}$ & 33.40 & ${ }^{-}-$ & $\begin{array}{c}- \\
32.77 \\
\end{array}$ & $\begin{array}{c}- \\
32.43 \\
\end{array}$ & $\begin{array}{c}- \\
32.13 \\
\end{array}$ & $\begin{array}{c}- \\
31.80 \\
\end{array}$ & $\begin{array}{c}- \\
31.43\end{array}$ & $\begin{array}{c}- \\
31.10\end{array}$ & $\begin{array}{c}- \\
30.80 \\
\end{array}$ & $\begin{array}{c}- \\
30.43 \\
\end{array}$ \\
\hline 9 & 0.88981 & $\begin{array}{c}- \\
33.00 \\
\end{array}$ & $\begin{array}{c}- \\
32.63 \\
\end{array}$ & $\begin{array}{c}- \\
32.30 \\
\end{array}$ & $\begin{array}{c}- \\
31.97 \\
\end{array}$ & $\begin{array}{c}- \\
31.60 \\
\end{array}$ & $\begin{array}{c}- \\
31.27 \\
\end{array}$ & $\begin{array}{c}- \\
30.93 \\
\end{array}$ & $\begin{array}{c}- \\
30.60 \\
\end{array}$ & $\begin{array}{c}- \\
30.20 \\
\end{array}$ & $\begin{array}{c}- \\
29.70\end{array}$ & $\begin{array}{c}- \\
29.10\end{array}$ \\
\hline 10 & 0.94783 & $\begin{array}{c}- \\
32.10 \\
\end{array}$ & $\begin{array}{c}- \\
31.70 \\
\end{array}$ & $\begin{array}{c}- \\
31.37 \\
\end{array}$ & 31.03 & $\begin{array}{c}- \\
30.80 \\
\end{array}$ & $\begin{array}{c}- \\
30.37 \\
\end{array}$ & $\begin{array}{c}- \\
29.93 \\
\end{array}$ & $\begin{array}{c}- \\
29.40 \\
\end{array}$ & 28.73 & 28.13 & 27.60 \\
\hline 11 & 1.00000 & $\begin{array}{c}- \\
31.27\end{array}$ & $\begin{array}{c}- \\
30.97\end{array}$ & $\begin{array}{c}- \\
30.60\end{array}$ & $\begin{array}{c}- \\
30.23 \\
\end{array}$ & $\begin{array}{c}- \\
29.83\end{array}$ & $\begin{array}{c}- \\
29.23\end{array}$ & $\begin{array}{c}- \\
28.53\end{array}$ & $\begin{array}{c}- \\
27.93\end{array}$ & $\begin{array}{c}- \\
27.43\end{array}$ & $\begin{array}{c}- \\
26.90\end{array}$ & $\begin{array}{c}- \\
26.40\end{array}$ \\
\hline
\end{tabular}

Table.6: Mole fraction of Acetonitrile in Toluene + Acetonitrile chemical mixtures and Output power at various temperatures (from $10^{\circ} \mathrm{C}$ to $60^{\circ} \mathrm{C}$ ), when operating wavelength of the source $820 \mathrm{~nm}$.

\begin{tabular}{|c|c|c|c|c|c|c|c|c|c|c|c|c|}
\hline \multirow{2}{*}{$\begin{array}{c}\text { S. } \\
\text { No. }\end{array}$} & \multirow{2}{*}{$\begin{array}{c}\text { Mole } \\
\text { fraction of } \\
\text { Acetonitrile }\end{array}$} & \multicolumn{11}{|c|}{ Output Power (dBm) at various temperatures } \\
\hline & & $10^{\circ} \mathrm{C}$ & $15^{\circ} \mathrm{C}$ & $20^{\circ} \mathrm{C}$ & $25^{\circ} \mathrm{C}$ & $3^{\circ} \mathrm{C}$ & $35^{\circ} \mathrm{C}$ & $40^{\circ} \mathrm{C}$ & $45^{\circ} \mathrm{C}$ & $\mathbf{5 0}^{\circ} \mathrm{C}$ & $\mathbf{5 5}^{\circ} \mathrm{C}$ & $60^{\circ} \mathrm{C}$ \\
\hline 1 & 0.00000 & $\begin{array}{c}- \\
46.83\end{array}$ & $\begin{array}{c}- \\
46.47\end{array}$ & $\begin{array}{c}- \\
46.07\end{array}$ & $\begin{array}{c}- \\
45.67\end{array}$ & $\begin{array}{c}- \\
45.23\end{array}$ & $\begin{array}{c}- \\
44.87\end{array}$ & $\begin{array}{c}- \\
44.47\end{array}$ & $\begin{array}{c}- \\
44.17\end{array}$ & $\begin{array}{c}- \\
43.80\end{array}$ & $\begin{array}{c}- \\
43.40\end{array}$ & $\begin{array}{c}- \\
43.03\end{array}$ \\
\hline 2 & 0.18321 & $\begin{array}{c}- \\
44.30\end{array}$ & $\begin{array}{c}- \\
43.90 \\
\end{array}$ & $\begin{array}{c}- \\
43.53 \\
\end{array}$ & $\begin{array}{c}- \\
43.20\end{array}$ & $\begin{array}{c}- \\
42.83 \\
\end{array}$ & $\begin{array}{c}- \\
42.47\end{array}$ & $\begin{array}{c}- \\
42.20\end{array}$ & $\begin{array}{c}- \\
41.90\end{array}$ & $\begin{array}{c}- \\
41.47\end{array}$ & $\begin{array}{c}- \\
41.03\end{array}$ & $\begin{array}{c}- \\
40.63 \\
\end{array}$ \\
\hline 3 & 0.33541 & $\begin{array}{c}- \\
42.33 \\
\end{array}$ & $\begin{array}{c}- \\
41.97 \\
\end{array}$ & $\begin{array}{c}- \\
41.60 \\
\end{array}$ & $\begin{array}{c}- \\
41.23 \\
\end{array}$ & $\begin{array}{c}- \\
40.87 \\
\end{array}$ & $\begin{array}{c}- \\
40.50 \\
\end{array}$ & $\begin{array}{c}- \\
40.10 \\
\end{array}$ & $\begin{array}{c}- \\
39.77 \\
\end{array}$ & $\begin{array}{c}- \\
39.27 \\
\end{array}$ & $\begin{array}{c}- \\
38.77 \\
\end{array}$ & ${ }^{-}-\overline{38}$ \\
\hline 4 & 0.46386 & $\begin{array}{c}- \\
40.67 \\
\end{array}$ & $\begin{array}{c}- \\
40.30 \\
\end{array}$ & $\begin{array}{c}- \\
39.90 \\
\end{array}$ & $\begin{array}{c}- \\
39.50 \\
\end{array}$ & $\begin{array}{c}- \\
39.13 \\
\end{array}$ & $\begin{array}{c}- \\
38.57 \\
\end{array}$ & $\begin{array}{c}- \\
38.13 \\
\end{array}$ & $\begin{array}{c}- \\
37.70 \\
\end{array}$ & $\begin{array}{c}- \\
37.43 \\
\end{array}$ & $\begin{array}{c}- \\
37.00 \\
\end{array}$ & $\begin{array}{c}- \\
36.57 \\
\end{array}$ \\
\hline 5 & 0.57372 & $\begin{array}{c}- \\
38.80\end{array}$ & $\begin{array}{c}- \\
38.46\end{array}$ & $\begin{array}{c}- \\
38.10\end{array}$ & $\begin{array}{c}- \\
37.77\end{array}$ & $\begin{array}{c}- \\
37.50\end{array}$ & $\begin{array}{c}- \\
37.00\end{array}$ & $\begin{array}{c}- \\
36.53\end{array}$ & $\begin{array}{c}- \\
36.30\end{array}$ & $\begin{array}{c}- \\
36.00\end{array}$ & $\begin{array}{c}- \\
35.67\end{array}$ & $\begin{array}{c}- \\
35.30\end{array}$ \\
\hline
\end{tabular}




\begin{tabular}{|c|c|c|c|c|c|c|c|c|c|c|c|c|}
\hline 6 & 0.66874 & $\begin{array}{c}- \\
37.50\end{array}$ & $\begin{array}{c}- \\
37.20\end{array}$ & $\begin{array}{c}- \\
36.70\end{array}$ & $\begin{array}{c}- \\
36.40\end{array}$ & $\begin{array}{c}- \\
36.13 \\
\end{array}$ & $\begin{array}{c}- \\
35.76\end{array}$ & $\begin{array}{c}- \\
35.40\end{array}$ & $\begin{array}{c}- \\
35.07\end{array}$ & $\begin{array}{c}- \\
34.73\end{array}$ & $\begin{array}{c}- \\
34.47\end{array}$ & $\begin{array}{c}- \\
34.13 \\
\end{array}$ \\
\hline 7 & 0.75175 & 36.53 & 36.27 & 35.83 & 35.47 & $\begin{array}{c}- \\
35.10\end{array}$ & 34.80 & 34.43 & 34.10 & 33.77 & 33.43 & 33.07 \\
\hline 8 & 0.82488 & $\begin{array}{c}- \\
35.30\end{array}$ & $\begin{array}{c}- \\
35.00\end{array}$ & $\begin{array}{c}- \\
34.67\end{array}$ & $\begin{array}{c}- \\
34.40\end{array}$ & $\begin{array}{c}- \\
34.03\end{array}$ & $\begin{array}{c}- \\
33.73\end{array}$ & $\begin{array}{c}- \\
33.40\end{array}$ & $\begin{array}{c}- \\
33.03\end{array}$ & $\begin{array}{c}- \\
32.70\end{array}$ & $\begin{array}{c}- \\
32.30\end{array}$ & $\begin{array}{c}- \\
31.90\end{array}$ \\
\hline 9 & 0.88981 & $\begin{array}{c}- \\
34.60\end{array}$ & 34.23 & $\begin{array}{c}- \\
33.90\end{array}$ & $\begin{array}{c}- \\
33.60\end{array}$ & $\begin{array}{c}- \\
33.20 \\
\end{array}$ & $\begin{array}{c}- \\
32.87\end{array}$ & $\begin{array}{c}- \\
32.53\end{array}$ & $\begin{array}{c}- \\
32.20\end{array}$ & $\begin{array}{c}- \\
31.80\end{array}$ & $\begin{array}{c}- \\
31.30\end{array}$ & $\begin{array}{c}- \\
30.70 \\
\end{array}$ \\
\hline 10 & 0.94783 & $\begin{array}{c}- \\
33.70 \\
\end{array}$ & $\begin{array}{c}- \\
33.30 \\
\end{array}$ & $\begin{array}{c}- \\
33.00 \\
\end{array}$ & $\begin{array}{c}- \\
32.63 \\
\end{array}$ & $\begin{array}{c}- \\
32.40 \\
\end{array}$ & $\begin{array}{c}- \\
31.97 \\
\end{array}$ & $\begin{array}{c}- \\
31.53 \\
\end{array}$ & $\begin{array}{c}- \\
31.00 \\
\end{array}$ & $\begin{array}{c}- \\
30.53 \\
\end{array}$ & $\begin{array}{c}- \\
30.03 \\
\end{array}$ & $\begin{array}{c}- \\
29.47 \\
\end{array}$ \\
\hline 11 & 1.00000 & $\begin{array}{c}- \\
32.97\end{array}$ & $\begin{array}{c}- \\
32.57\end{array}$ & $\begin{array}{c}- \\
32.20\end{array}$ & $\begin{array}{c}- \\
31.83\end{array}$ & $\begin{array}{c}- \\
31.43\end{array}$ & $\begin{array}{c}- \\
30.83\end{array}$ & $\begin{array}{c}- \\
30.30\end{array}$ & $\begin{array}{c}- \\
29.80\end{array}$ & $\begin{array}{c}- \\
29.27\end{array}$ & $\begin{array}{c}- \\
28.83\end{array}$ & $\begin{array}{c}- \\
28.27\end{array}$ \\
\hline
\end{tabular}

Table.7: Mole fraction of Acetonitrile in Toluene + Acetonitrile chemical mixtures and Output power at various temperatures (from $10^{\circ} \mathrm{C}$ to $60^{\circ} \mathrm{C}$ ), when operating wavelength of the source $850 \mathrm{~nm}$.

\begin{tabular}{|c|c|c|c|c|c|c|c|c|c|c|c|c|}
\hline \multirow{2}{*}{$\begin{array}{c}\text { S. } \\
\text { No. }\end{array}$} & \multirow{2}{*}{$\begin{array}{c}\text { Mole } \\
\text { fraction of } \\
\text { Acetonitrile } \\
\end{array}$} & \multicolumn{11}{|c|}{ Output Power $(\mathrm{dBm})$ at various temperatures } \\
\hline & & $10^{\circ} \mathrm{C}$ & $15^{\circ} \mathrm{C}$ & $20^{\circ} \mathrm{C}$ & $25^{\circ} \mathrm{C}$ & $3^{\circ}{ }^{\circ} \mathrm{C}$ & $\mathbf{3 5}^{\circ} \mathrm{C}$ & $40^{\circ} \mathrm{C}$ & $\mathbf{4 5}^{\circ} \mathrm{C}$ & $\mathbf{5 0}^{\circ} \mathrm{C}$ & $55^{\circ} \mathrm{C}$ & $60^{\circ} \mathrm{C}$ \\
\hline 1 & 0.00000 & $\begin{array}{c}- \\
47.23 \\
\end{array}$ & $\begin{array}{c}- \\
46.83 \\
\end{array}$ & $\begin{array}{c}- \\
46.43 \\
\end{array}$ & $\begin{array}{c}- \\
46.03 \\
\end{array}$ & $\begin{array}{c}- \\
45.60\end{array}$ & $\begin{array}{c}- \\
45.23\end{array}$ & $\begin{array}{c}- \\
44.83 \\
\end{array}$ & 44.53 & $\begin{array}{c}- \\
44.17\end{array}$ & $\begin{array}{c}- \\
43.77 \\
\end{array}$ & 43.40 \\
\hline 2 & 0.18321 & $\begin{array}{c}- \\
44.67 \\
\end{array}$ & $\begin{array}{c}- \\
44.27 \\
\end{array}$ & $\begin{array}{c}- \\
43.90 \\
\end{array}$ & $\begin{array}{c}- \\
43.57 \\
\end{array}$ & $\begin{array}{c}- \\
43.20 \\
\end{array}$ & $\begin{array}{c}- \\
42.83 \\
\end{array}$ & $\begin{array}{c}- \\
42.57 \\
\end{array}$ & $\begin{array}{c}- \\
42.27 \\
\end{array}$ & $\begin{array}{c}- \\
41.83 \\
\end{array}$ & $\begin{array}{c}- \\
41.40 \\
\end{array}$ & $\begin{array}{c}- \\
41.00 \\
\end{array}$ \\
\hline 3 & 0.33541 & $\begin{array}{c}- \\
42.70 \\
\end{array}$ & $\begin{array}{c}- \\
42.33 \\
\end{array}$ & $\begin{array}{c}- \\
41.97 \\
\end{array}$ & $\begin{array}{c}- \\
41.60 \\
\end{array}$ & $\begin{array}{c}- \\
41.23 \\
\end{array}$ & $\begin{array}{c}- \\
40.87 \\
\end{array}$ & $\begin{array}{c}- \\
40.47 \\
\end{array}$ & $\begin{array}{c}- \\
40.13 \\
\end{array}$ & $\begin{array}{c}- \\
39.63 \\
\end{array}$ & $\begin{array}{c}- \\
39.13 \\
\end{array}$ & $\begin{array}{c}- \\
38.73 \\
\end{array}$ \\
\hline 4 & 0.46386 & $\begin{array}{c}- \\
41.07\end{array}$ & $\begin{array}{c}- \\
40.67 \\
\end{array}$ & $\begin{array}{c}- \\
40.27 \\
\end{array}$ & $\begin{array}{c}- \\
39.87 \\
\end{array}$ & $\begin{array}{c}- \\
39.50 \\
\end{array}$ & $\begin{array}{c}- \\
38.93 \\
\end{array}$ & $\begin{array}{c}- \\
38.50\end{array}$ & $\begin{array}{c}- \\
38.07 \\
\end{array}$ & $\begin{array}{c}- \\
37.80 \\
\end{array}$ & $\begin{array}{c}- \\
37.37 \\
\end{array}$ & $\begin{array}{c}- \\
36.93 \\
\end{array}$ \\
\hline 5 & 0.57372 & $\begin{array}{c}- \\
39.17 \\
\end{array}$ & $\begin{array}{c}- \\
38.83 \\
\end{array}$ & $\begin{array}{c}- \\
38.47 \\
\end{array}$ & $\begin{array}{c}- \\
38.13 \\
\end{array}$ & $\begin{array}{c}- \\
37.87 \\
\end{array}$ & $\begin{array}{c}- \\
37.37 \\
\end{array}$ & $\begin{array}{c}- \\
36.90 \\
\end{array}$ & $\begin{array}{c}- \\
36.67 \\
\end{array}$ & $\begin{array}{c}- \\
36.37 \\
\end{array}$ & $\begin{array}{c}- \\
36.03 \\
\end{array}$ & $\begin{array}{c}- \\
35.67 \\
\end{array}$ \\
\hline 6 & 0.66874 & $\begin{array}{c}- \\
37.87 \\
\end{array}$ & $\begin{array}{c}- \\
37.57 \\
\end{array}$ & $\begin{array}{c}- \\
37.07 \\
\end{array}$ & $\begin{array}{c}- \\
36.77 \\
\end{array}$ & $\begin{array}{c}- \\
36.50 \\
\end{array}$ & $\begin{array}{c}- \\
36.13 \\
\end{array}$ & $\begin{array}{c}- \\
35.77\end{array}$ & $\begin{array}{c}- \\
35.43 \\
\end{array}$ & $\begin{array}{c}- \\
35.10\end{array}$ & $\begin{array}{c}- \\
34.83 \\
\end{array}$ & $\begin{array}{c}- \\
34.50 \\
\end{array}$ \\
\hline 7 & 0.75175 & $\begin{array}{c}- \\
36.90\end{array}$ & $\begin{array}{c}- \\
36.63\end{array}$ & $\begin{array}{c}- \\
36.23\end{array}$ & $\begin{array}{c}- \\
35.83\end{array}$ & $\begin{array}{c}- \\
35.47\end{array}$ & $\begin{array}{c}- \\
35.17\end{array}$ & $\begin{array}{c}- \\
34.80\end{array}$ & $\begin{array}{c}- \\
34.47\end{array}$ & $\begin{array}{c}- \\
34.13\end{array}$ & $\begin{array}{c}- \\
33.80\end{array}$ & $\begin{array}{c}- \\
33.43\end{array}$ \\
\hline 8 & 0.82488 & $\begin{array}{c}- \\
35.67 \\
\end{array}$ & $\begin{array}{c}- \\
35.37 \\
\end{array}$ & $\begin{array}{c}- \\
35.03 \\
\end{array}$ & $\begin{array}{c}- \\
34.77 \\
\end{array}$ & $\begin{array}{c}- \\
34.40 \\
\end{array}$ & $\begin{array}{c}- \\
34.10 \\
\end{array}$ & $\begin{array}{c}- \\
33.77 \\
\end{array}$ & $\begin{array}{c}- \\
33.40 \\
\end{array}$ & $\begin{array}{c}- \\
33.07 \\
\end{array}$ & $\begin{array}{c}- \\
32.67 \\
\end{array}$ & $\begin{array}{c}- \\
32.27 \\
\end{array}$ \\
\hline 9 & 0.88981 & $\begin{array}{c}- \\
34.97 \\
\end{array}$ & $\begin{array}{c}- \\
34.60 \\
\end{array}$ & $\begin{array}{c}- \\
34.27 \\
\end{array}$ & $\begin{array}{c}- \\
33.97 \\
\end{array}$ & $\begin{array}{c}- \\
33.57 \\
\end{array}$ & $\begin{array}{c}- \\
33.23 \\
\end{array}$ & $\begin{array}{c}- \\
32.90\end{array}$ & $\begin{array}{c}- \\
32.57 \\
\end{array}$ & $\begin{array}{c}- \\
32.17 \\
\end{array}$ & $\begin{array}{c}- \\
31.67 \\
\end{array}$ & $\begin{array}{c}- \\
31.07 \\
\end{array}$ \\
\hline 10 & 0.94783 & $\begin{array}{r}- \\
34.07\end{array}$ & 33.67 & 33.37 & 33.03 & $\begin{array}{c}- \\
32.77\end{array}$ & 32.33 & 31.90 & 31.37 & 30.90 & 30.40 & 29.83 \\
\hline 11 & 1.00000 & $\begin{array}{c}- \\
33.33 \\
\end{array}$ & $\begin{array}{c}- \\
32.93 \\
\end{array}$ & $\begin{array}{c}- \\
32.57 \\
\end{array}$ & $\begin{array}{c}- \\
32.20\end{array}$ & $\begin{array}{c}- \\
31.80 \\
\end{array}$ & $\begin{array}{c}- \\
31.20\end{array}$ & $\begin{array}{c}- \\
30.67 \\
\end{array}$ & $\begin{array}{c}- \\
30.17\end{array}$ & $\begin{array}{c}- \\
29.63\end{array}$ & $\begin{array}{c}- \\
29.20\end{array}$ & 28.63 \\
\hline
\end{tabular}

\section{RESULTS AND DISCUSSION}

The fractional value of one liquid in the mixture of liquids influences the refractive index of the mixture. The studies of mole fractions of all the mixtures and the refractive index studies of all mixtures at different temperatures thus enable to form the relationship between one on another, which are presented in the form of graph [fig.2]. As there is a direct relationship between dielectric constant and refractive index and hence the mole fraction, a graph is plotted between dielectric constant and mole fraction [fig.3]. 


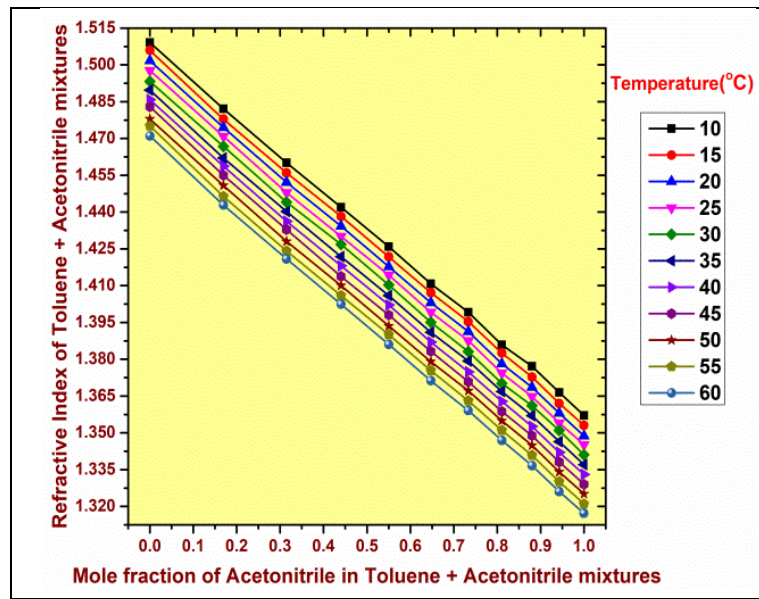

Fig.2: Relation between Mole fraction of Acetonitrile in Toluene + Acetonitrile mixtures and Refractive index.

Therefore, the presence of percentage of concentration of Acetonitrile in the mixture of Toluene + Acetonitrile changes the refractive index and hence dielectric constant of the liquid mixture, the corresponding results

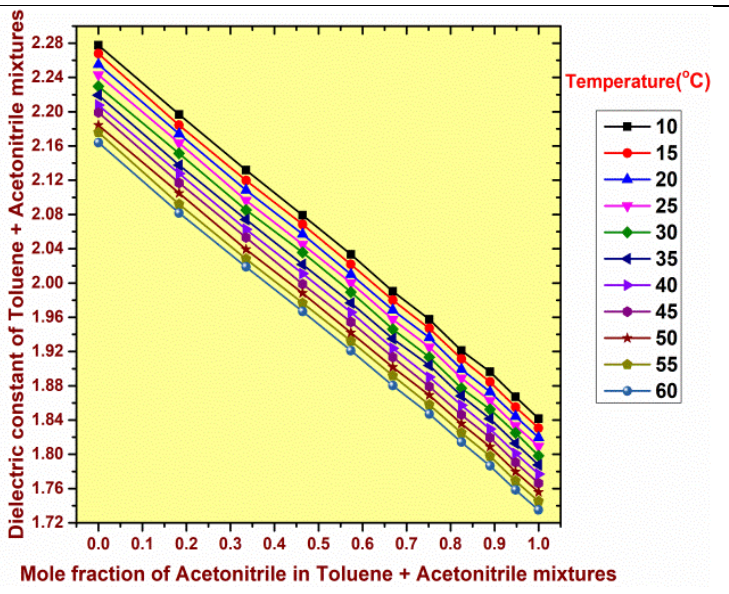

Fig.3: Relation between Mole fraction of Acetonitrile in Toluene + Acetonitrile mixtures and Dielectric constant.

connecting the concentration percentage of Acetonitrile in the mixtures, refractive index and hence the dielectric constant of the mixtures at different temperatures were presented in graphs [fig. 4,5$]$.

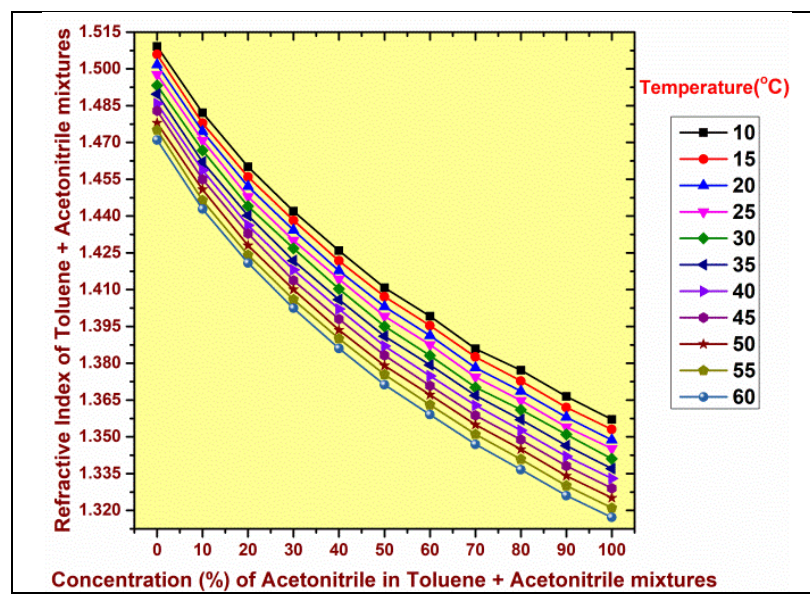

Fig.4: Relation between Concentration (\%) of Acetonitrile in Toluene + Acetonitrile mixtures and Refractive index.

The refractive index changes of the mixtures were observed to be dramatic with increase in temperature of the mixtures. As the temperature increases, the refractive index of mixtures appears decreasing and results were

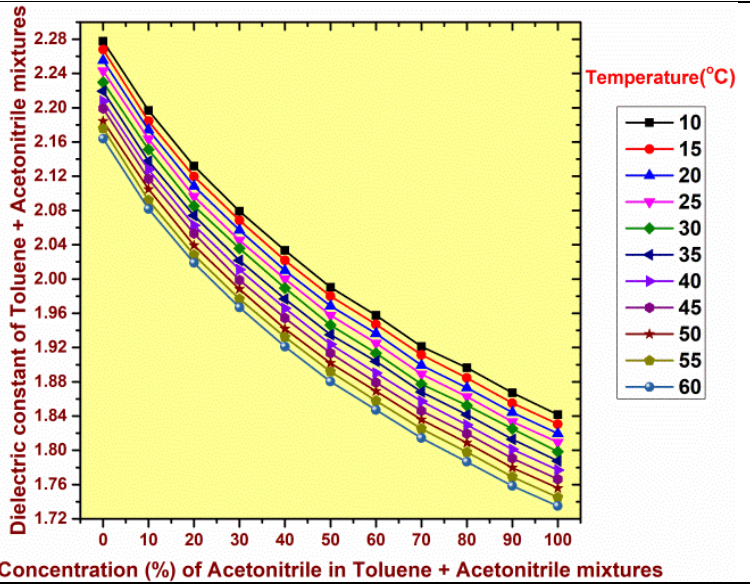

Fig.5: Relation between Concentration (\%) of Acetonitrile in Toluene + Acetonitrile mixtures and Dielectric constant.

shown graphically [fig.6]. As the refractive index and dielectric constant are related proportional to one on another, it was observed that the dielectric constant also decreases with raise in temperature and was shown pictorially [fig.7]. 


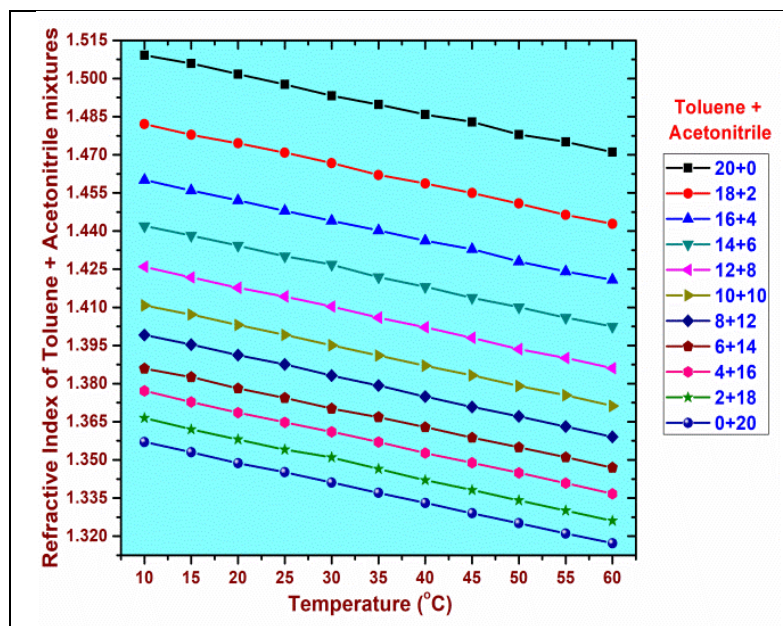

Fig.6: Relation between Temperature and Refractive index of Toluene + Acetonitrile mixtures.

The dependence between the mole fraction of Acetonitrile in liquid mixtures and the temperature dependent refractive index of the mixtures helps to plot a 3dimentional graph to present the mutual relationships of the three parameters i.e. mole fraction, refractive index

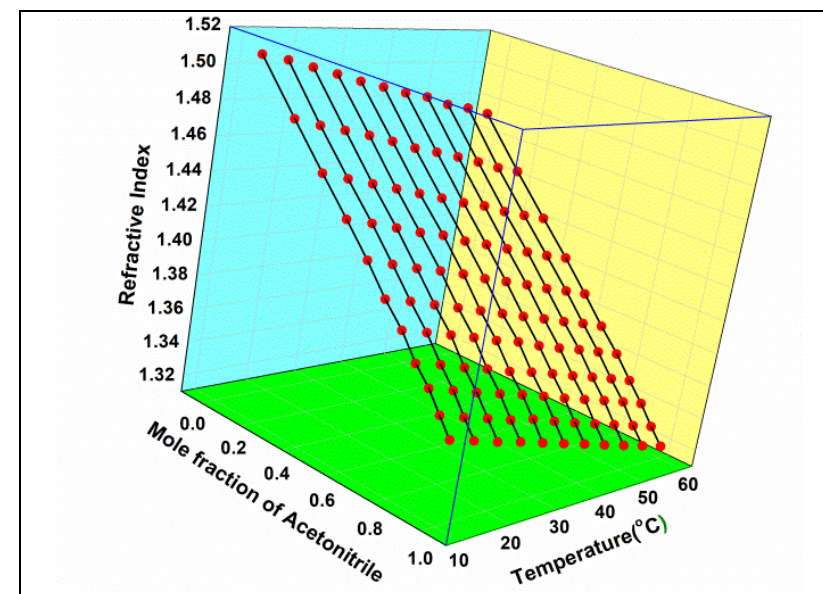

Fig.8: Relation among Mole fraction of Acetonitrile in Toluene + Acetonitrile mixtures, Refractive index and Temperature.

It is interesting to relate the mole fraction with the temperature dependent dielectric constant in a 3 dimentional graph [fig.10]. The raise in temperature decreases the dielectric constant of the mixtures and which was in turn depends on the presence of mole

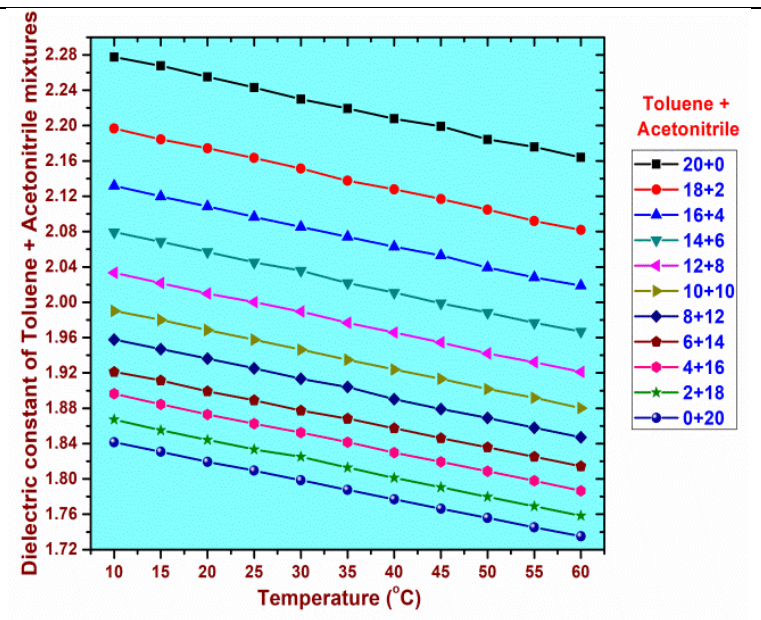

Fig.7: Relation between Temperature and Dielectric constant of Toluene + Acetonitrile mixtures.

and temperature [fig.8]. Thus, the percentage concentration of the presence of Acetonitrile in the mixtures influences the temperature dependent refractive index, and hence the mutual dependence of concentration, refractive index and temperature are presented in a 3dimentional graph [fig.9].

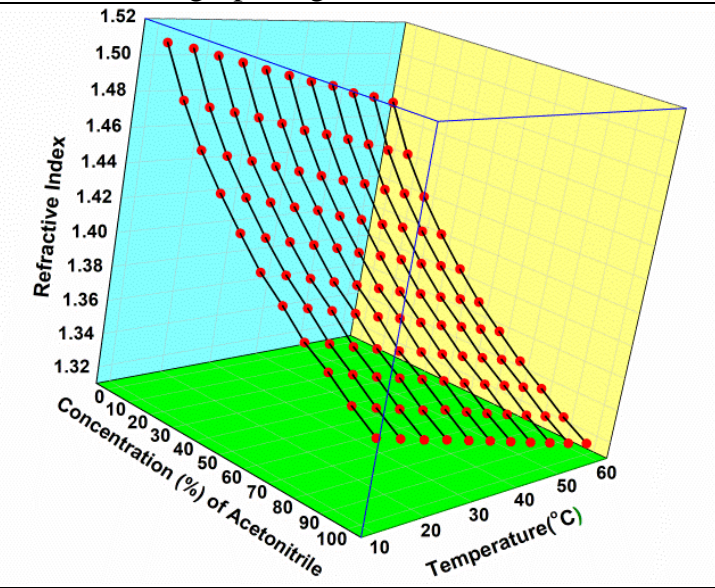

Fig.9: Relation among Concentration (\%) of Acetonitrile in Toluene + Acetonitrile mixtures, Refractive index and Temperature.

fractional values of Acetonitrile in the liquid mixtures. As the concentration of Acetonitrile increases, the dielectric constant of the mixture decreases, which variation was further changed by the raise in temperature and the relationships were presented in 3dimentional graph [fig.11]. 


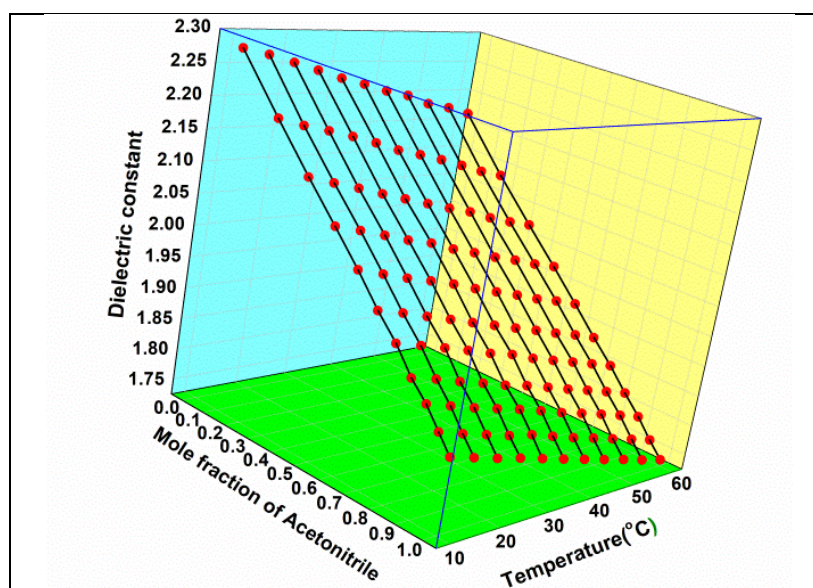

Fig.10: Relation among Mole fraction of Acetonitrile in Toluene + Acetonitrile mixtures, Dielectric constant and Temperature.

At a particular wavelength of source $(630 \mathrm{~nm})$, the light reaching the detector by changing the mole fractions of Acetonitrile in the mixtures and maintaining the mixtures at various temperatures was studied and relationships are

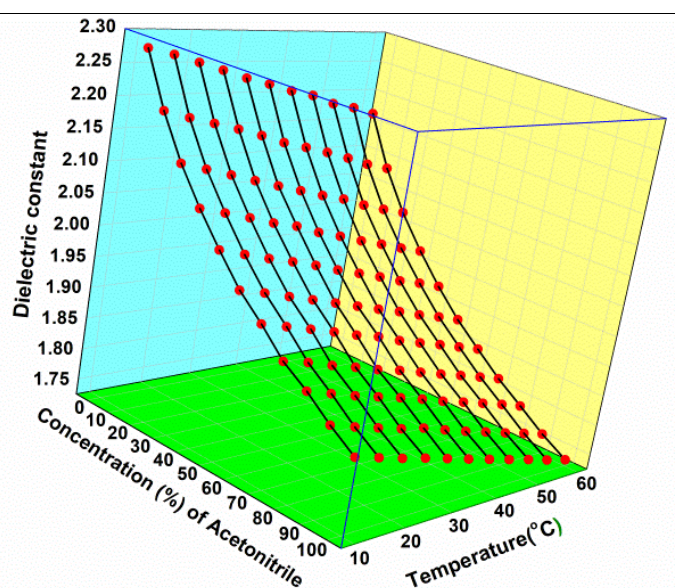

Fig.11: Relation among Concentration (\%) of Acetonitrile in Toluene + Acetonitrile mixtures, Dielectric constant and Temperature.

presented in the form of graph [fig.12]. The above study of was conducted by repeating the experiment by varying the wavelengths of the source as $660 \mathrm{~nm}, 820 \mathrm{~nm}$ and 850 nm [fig.13-15].

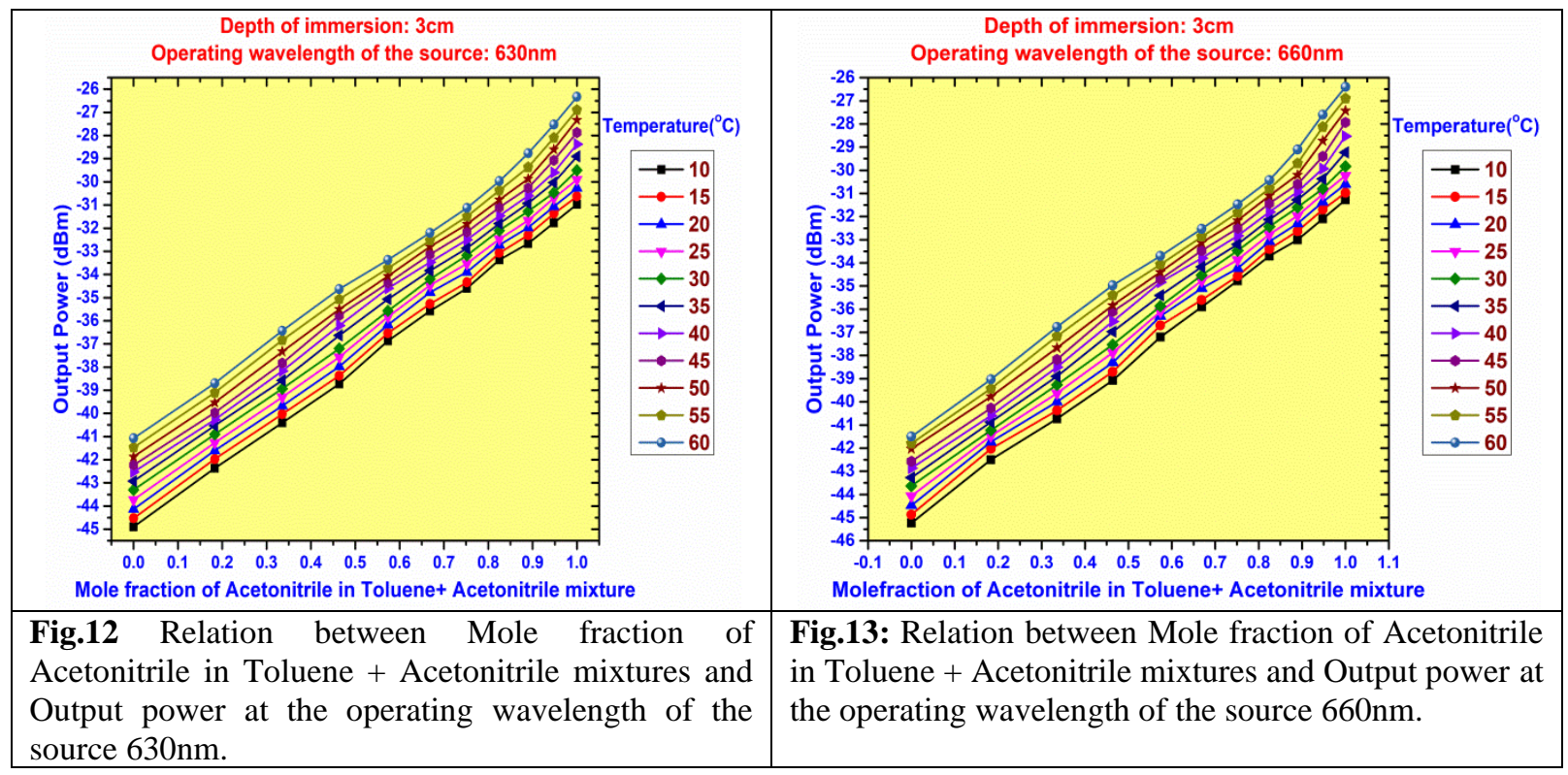




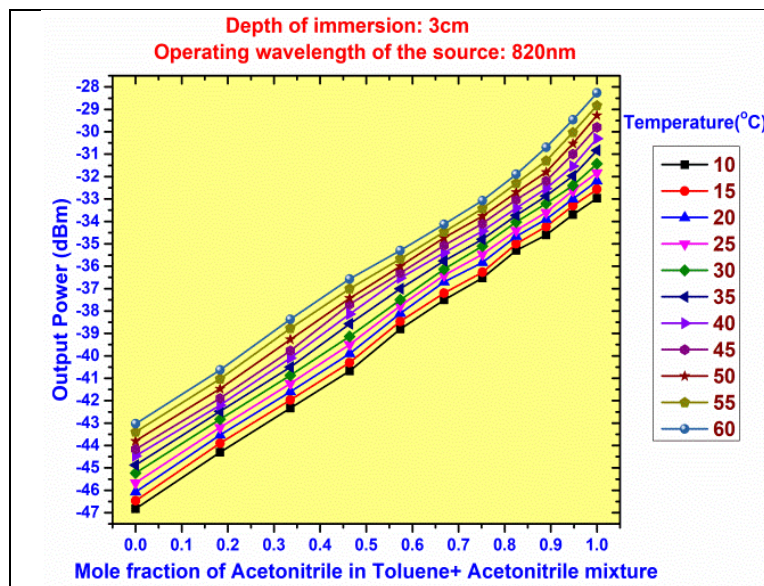

Fig.14: Relation between Mole fraction of Acetonitrile in Toluene + Acetonitrile mixtures and Output power at operating wavelength of the source $820 \mathrm{~nm}$.

The output powers for all the mixtures were changing with respect to percentage presence of Acetonitrile in the liquid mixtures and at different temperatures corresponding to a particular wavelength $(630 \mathrm{~nm})$ of

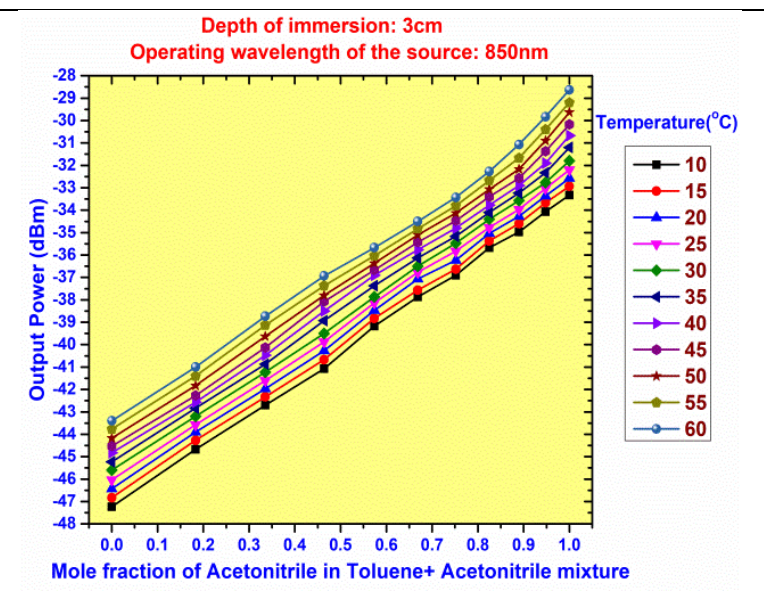

Fig.15: Relation between Mole fraction of Acetonitrile in Toluene + Acetonitrile mixtures and Output power at the operating wavelength of the source $850 \mathrm{~nm}$.

operation. The study was taken up by repeating the above procedure employing $660 \mathrm{~nm}, 820 \mathrm{~nm}$ and $850 \mathrm{~nm}$ of source wavelengths, and the trend was observed to be same [fig.16-19].

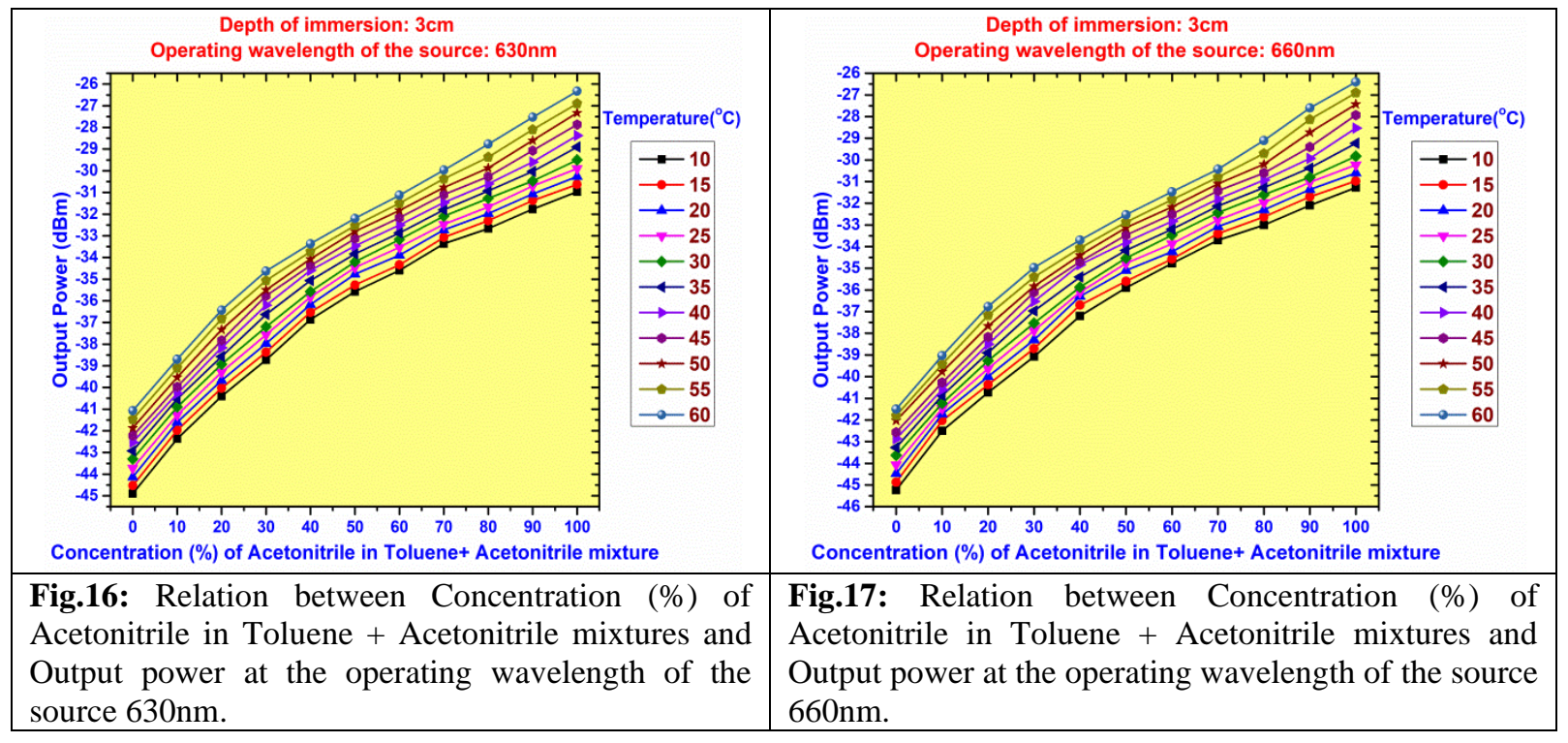




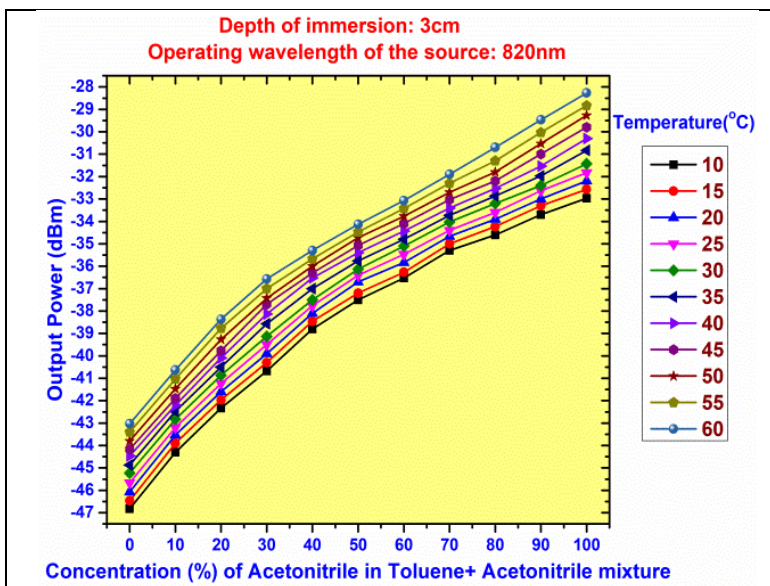

Fig. 18: Relation between Concentration (\%) of Acetonitrile in Toluene + Acetonitrile mixtures and Output power at operating wavelength of the source $820 \mathrm{~nm}$.

The chemical mixture around the glass rod as an analyte acts as a liquid cladding in transmitting the light from the source to detector. The light while travelling along the Ushaped glass rod, suffers loss due to the removal of the cladding. Due to which the higher order modes of the light escape out of the rod as leaky radiation modes. The leakage of light into the liquid is a function of refractive index of the liquid (analyte) cladding. As the refractive

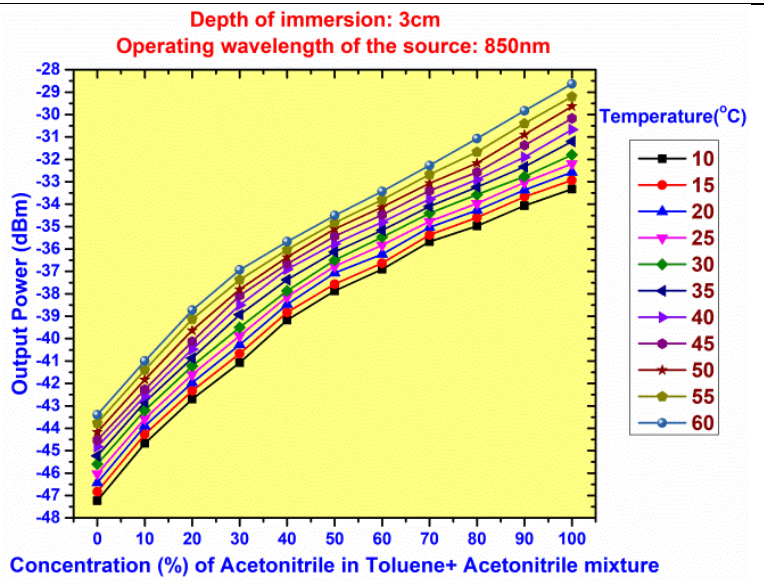

Fig.19: Relation between Concentration (\%) of Acetonitrile in Toluene + Acetonitrile mixtures and Output power at the operating wavelength of the source $850 \mathrm{~nm}$.

index of the liquid cladding increases, the absorption of higher modes into the liquid and hence the less amount of output power observed in the detector. As the refractive index of liquid is temperature dependent, the output powers also various in accordance with the temperature variation. This phenomenon was represented graphically and observed that the output power increases with increase in temperature of the mixture at all wavelengths i.e. $630 \mathrm{~nm}, 660 \mathrm{~nm}, 820 \mathrm{~nm}$ and $850 \mathrm{~nm}$ [fig. 20-23].

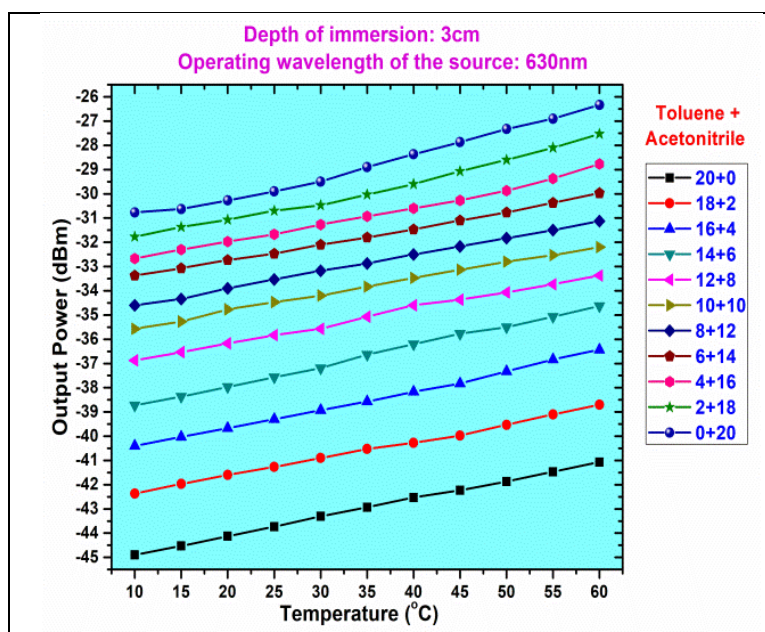

Fig.20: Relation between Temperature and Output power of Toluene + Acetonitrile mixtures at the operating wavelength of the source $630 \mathrm{~nm}$

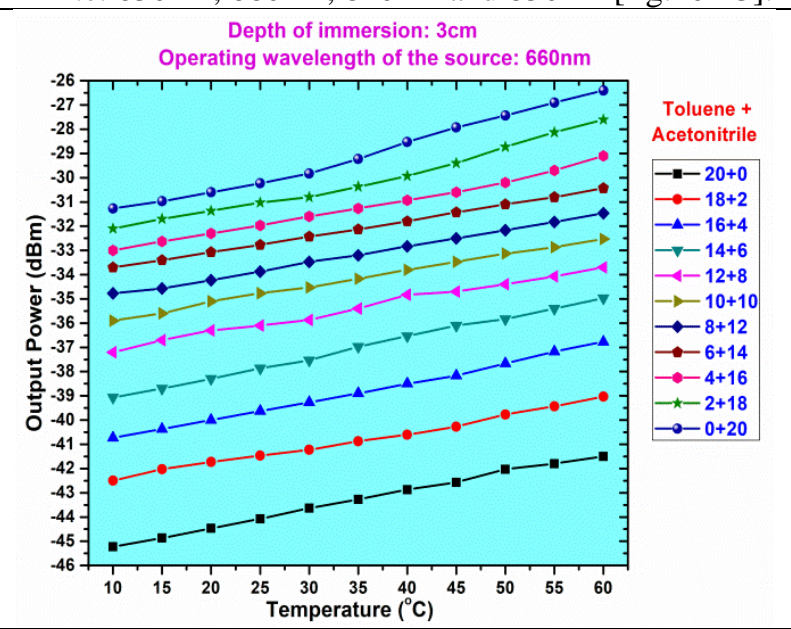

Fig.21: Relation between Temperature and Output power of Toluene + Acetonitrile mixtures at the operating wavelength of the source $660 \mathrm{~nm}$ 


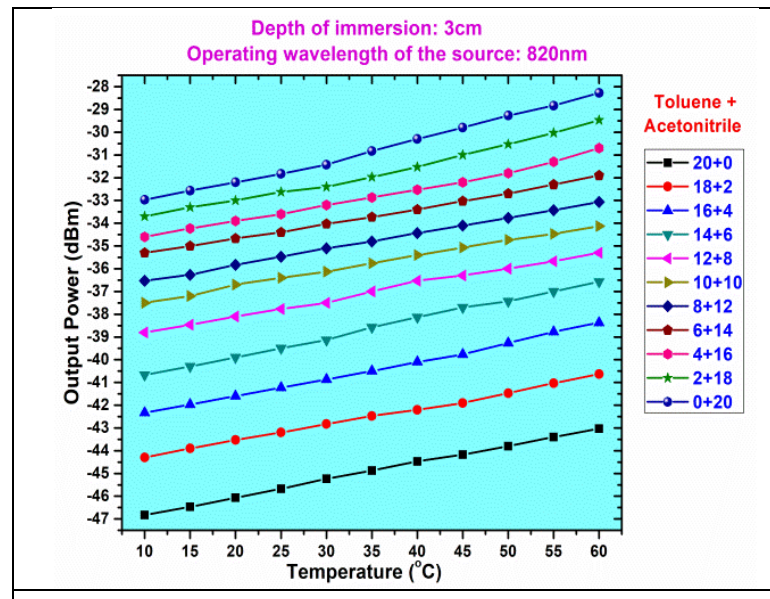

Fig.22: Relation between Temperature and Output power of Toluene + Acetonitrile mixtures at the operating wavelength of the source $820 \mathrm{~nm}$

The temperature dependent variation of refractive index influences the power reaching the detector. As the temperature of the analyte around the glass rod increases, the refractive index of analyte decreases and hence the

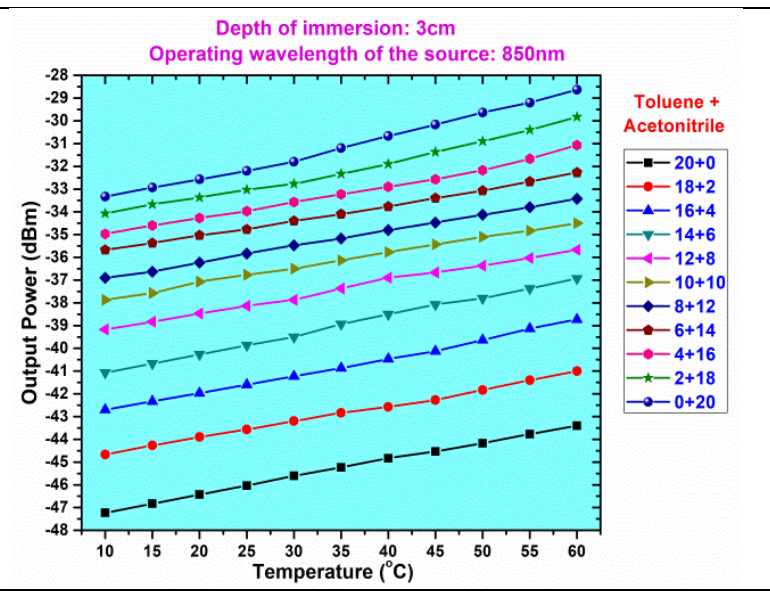

Fig.23: Relation between Temperature and Output power of Toluene + Acetonitrile mixtures at the operating wavelength of the source $850 \mathrm{~nm}$

power reaching the detector increases. Thus, the change in the temperature, changes the refractive and hence the corresponding change in the output power and the results are shown in figures [fig.24-27].

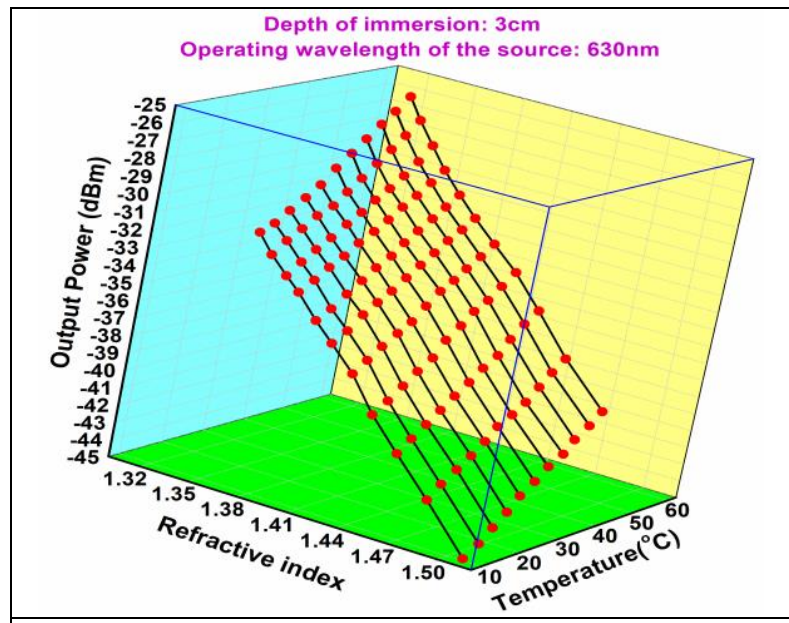

Fig.24: Relation between Refractive index, Output Power and Temperature of Toluene + Acetonitrile at the operating wavelength of the source $630 \mathrm{~nm}$

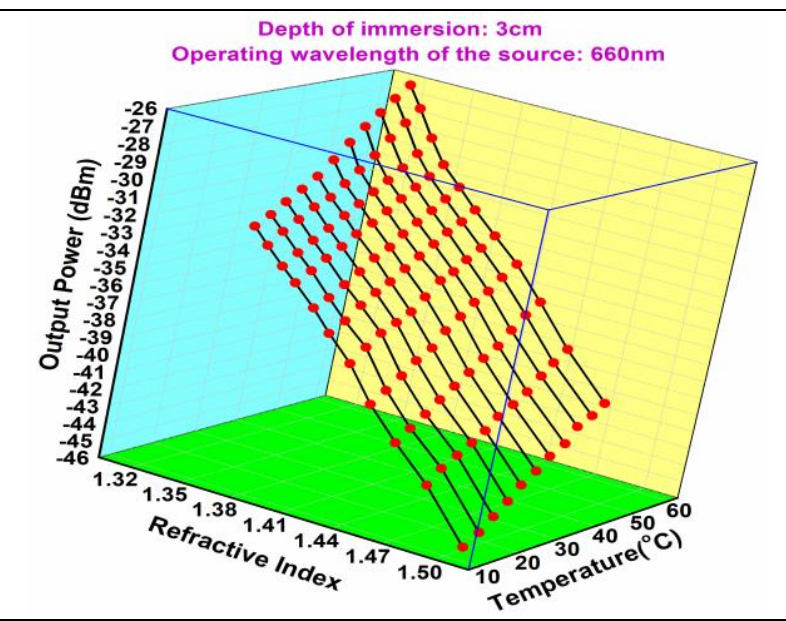

Fig.25: Relation between Refractive index, Output Power and Temperature of Toluene + Acetonitrile at the operating wavelength of the source $660 \mathrm{~nm}$ 


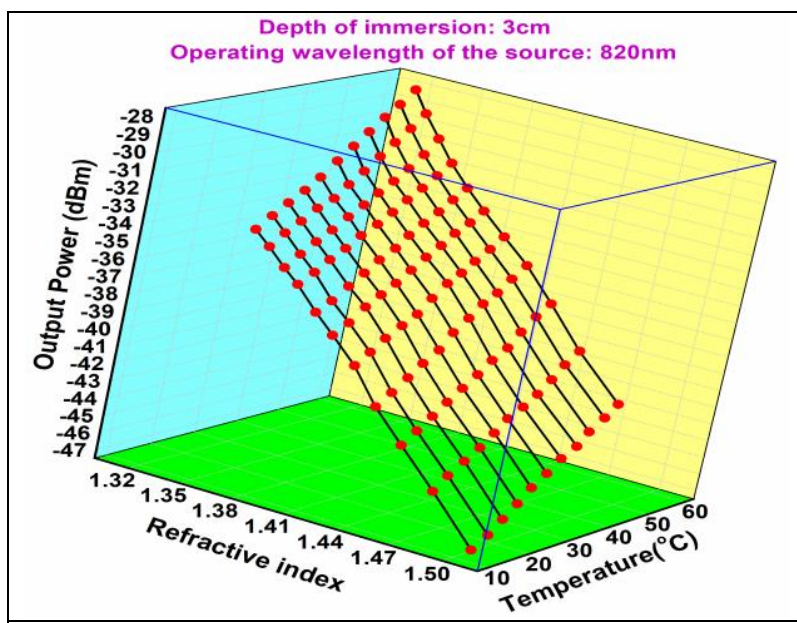

Fig.26: Relation between Refractive index, Output Power and Temperature of Toluene + Acetonitrile at the operating wavelength of the source $820 \mathrm{~nm}$

As the dielectric constant of the liquid is a function of refractive index and has direct proportionality, the dielectric constant also influences the output power of the sensor. Thus, as the temperature increases, the dielectric constant decreases, which in turn increases the output

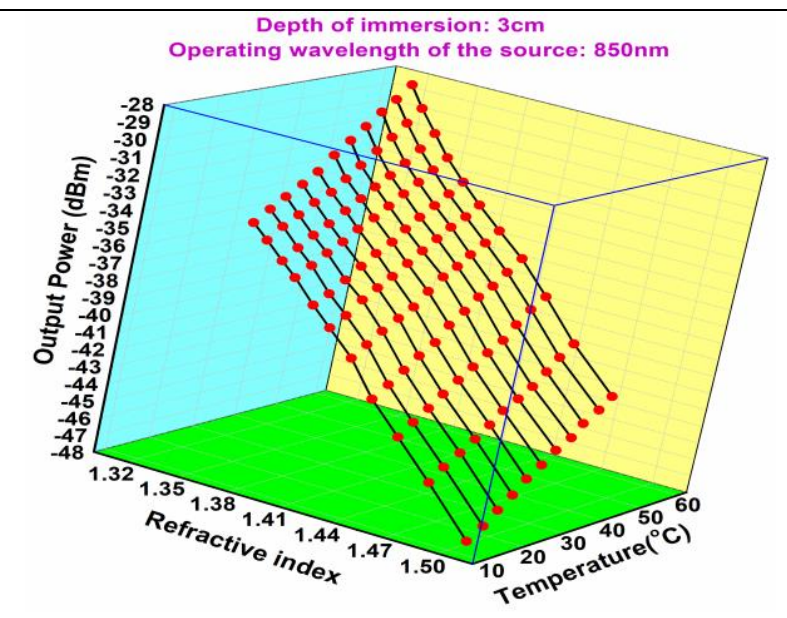

Fig.27: Relation between Refractive index, Output Power and Temperature of Toluene + Acetonitrile at the operating wavelength of the source $850 \mathrm{~nm}$

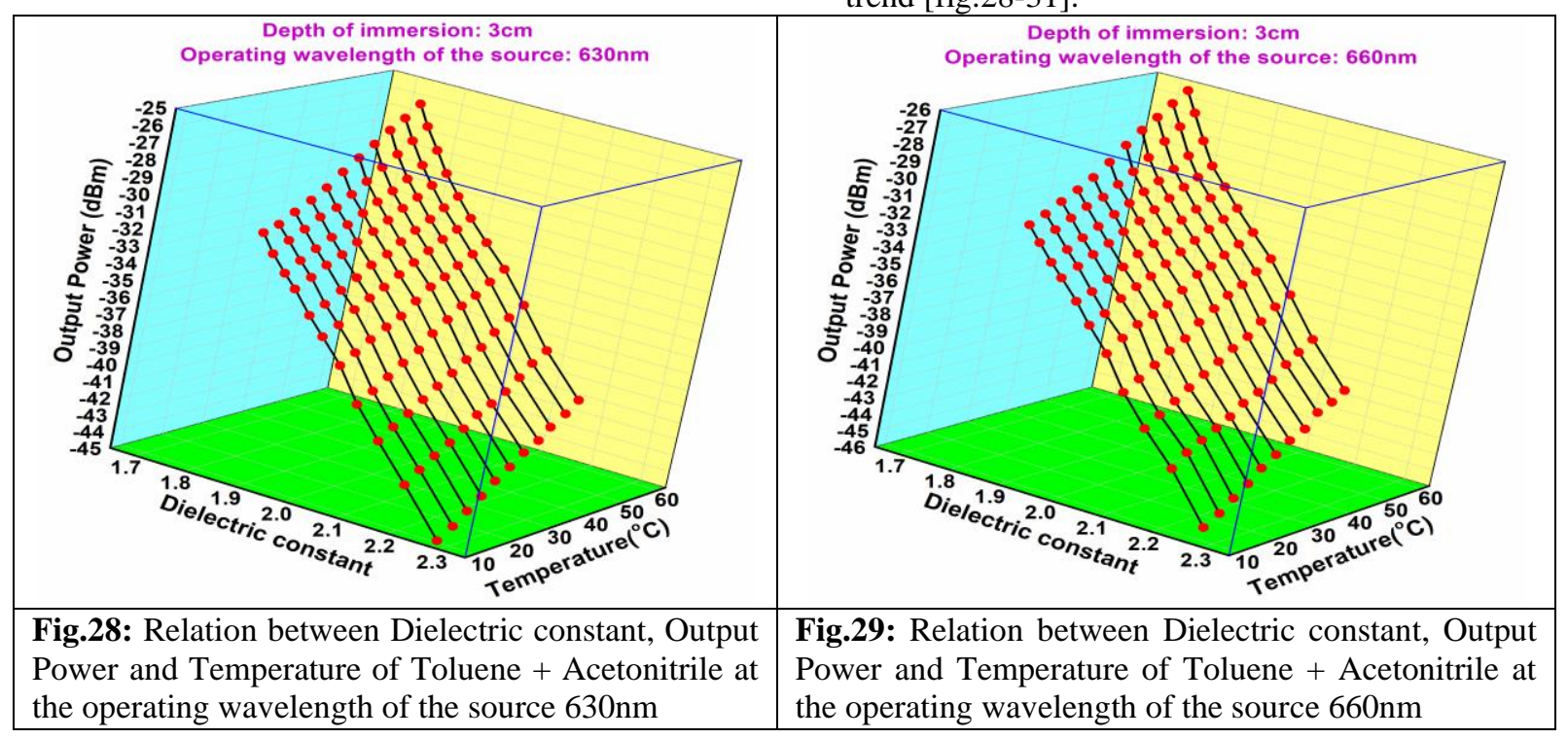
power. The variation of output power due to the temperature dependent dielectric constant of the mixture was observed to be increased with increase in the temperature of the mixture at the source wavelengths of $630 \mathrm{~nm}, 660 \mathrm{~nm}, 820 \mathrm{~nm} \& 850 \mathrm{~nm}$ and following same trend [fig.28-31].

Power and 1 the operating wavelength of the source $660 \mathrm{~nm}$ 


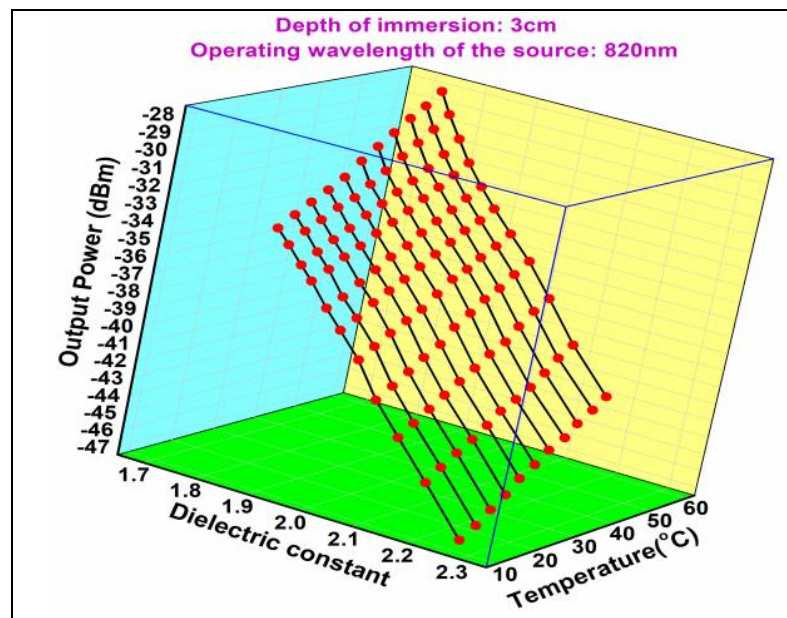

Fig.30: Relation between Dielectric constant, Output Power and Temperature of Toluene + Acetonitrile at the operating wavelength of the source $820 \mathrm{~nm}$

\section{CONCLUSION}

In the present work, a refractometer was calibrated and developed to operate between $10^{\circ} \mathrm{C}$ to $60^{\circ} \mathrm{C}$ and at wavelength of $630 \mathrm{~nm}, 660 \mathrm{~nm}, 820 \mathrm{~nm} \& 850 \mathrm{~nm}$ to determine the refractive index of certain dark and transparent liquids. A set of two binary liquids i.e. Toluene and Acetonitrile has been selected as test liquids in the calibration of the sensor. The data corresponding to output powers related to all the mixtures around the Ushaped glass rod by changing the refractive index with respect to temperature at the wavelengths of $630 \mathrm{~nm}$, $660 \mathrm{~nm}, 820 \mathrm{~nm}$ and $850 \mathrm{~nm}$ have been obtained. As the dielectric constant values of liquids have the direct consequence on the refractive index, the study on output power variation on the dielectric constant was under taken to show that, as the dielectric constant increases, the output power decreases. Thus, the present sensor is a unique and versatile refractive index sensor operating at $630 \mathrm{~nm}, 660 \mathrm{~nm}, 820 \mathrm{~nm} \& 850 \mathrm{~nm}$ and at the temperature range of $10^{\circ} \mathrm{C}$ to $60^{\circ} \mathrm{C}$ which was proved to be superior in many aspects comparing with conventional sensors.

\section{REFERENCES}

[1] Rajamani, A.S.; Divagar, M.; Sai, V.V.R. Plastic fiber optic sensor for continuous liquid level monitoring. Sens. Actuator A 2019, 296, 192-199.

[2] Fatemi, F.K.; Bashkansky, M. Generation of hollow beams by using a binary spatial light modulator. Opt. Lett. 2006, 31, 864-866.

[3] Lu, L.; Jiang, Z.; Hu, Y.; Zhou, H.; Chen, Z. A portable optical fiber SPR temperature sensor based on a smart-phone. Opt. Express 2019, 27, 25420.

[4] Gowri, A.; Rajamani, A.S.; Ramakrishna, B.; Sai, V.V.R. U-bent plastic optical fiber probes as refractive

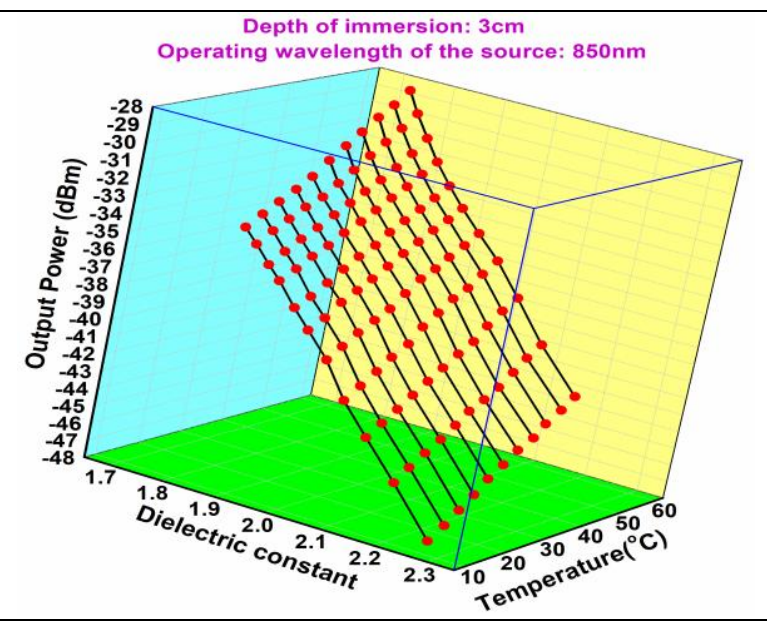

Fig.31: Relation between Dielectric constant, Output Power and Temperature of Toluene + Acetonitrile at the operating wavelength of the source $850 \mathrm{~nm}$

index based fat sensor for milk quality monitoring. Opt. Fiber Technol. 2019, 47, 15-20.

[5] Xu, Y.; Lu, P.; Chen, L.; Bao, X. Recent Developments in Micro-Structured Fiber Optic Sensors. Fibers 2017, 5, 3.

[6] Zhou, W.; Zhou, Y.; Albert, J. A true fiber optic refractometer. Laser Photonics Rev. 2017, 11, 1600157.

[7] Zhang, X.; Xie, L.; Zhang, X.; Peng, W. Optimization of long-period grating-based refractive index sensor by bent-fiber interference. Appl. Opt. 2015, 54, 9152-9156.

[8] Bhatia, N.; John, J. Multimode interference devices with single-mode-multimode-multimode fiber structure. Appl. Opt. 2014, 53, 5179-5186.

[9] Divagar, M.; Gowri, A.; John, S.; Sai, V.V.R. Graphene oxide coated U-bent plastic optical fiber based chemical sensor for organic solvents. Sens. Actuator B 2018, 262, 1006-1012.

[10] Gowri, A.; Sai, V.V.R. Development of LSPR based U-bent plastic optical fiber sensor. Sens. Actuator B 2016, 230, 536-543.

[11] Arrue, F.; Jiménez, G.; Aldabaldetreku, G.; Durana, J.; Zubia, M.; Lomer, J. Analysis of the use of tapered graded-index polymer optical fibers for refractive-index sensors. Opt. Express 2008, 16, 16616-16631.

[12] Gong, W.; Jiang, S.; Li, Z.; Li, C.; Zhang, C. Experimental and theoretical investigation for surface plasmon resonance biosensor based on graphene/Au film/D-POF. Opt. Express 2019, 27, 3483.

[13] Chen, C.H.; Tsao, T.C.; Tang, J.L.; Wu, W.T. A Multi-D-Shaped Optical Fiber for Refractive Index Sensing. Sensors 2010, 10, 4794-4804.

[14] Shin, J.; Park, J. Plastic optical fiber refractive index sensor employing an in-line submillimeter hole. IEEE Photonics Technol. Lett. 2013, 25, 1882-1884. 
[15] Feng, D.; Liu, G.; Liu, X.; Jiang, M.; Sui, Q. Refractive index sensor based on plastic optical fiber with tapered structure. Appl. Opt. 2014, 53, 2007-2011.

[16] Teng, C.; Deng, H.; Liu, H.; Yang, H.; Yuan, L.; Zheng, J.; Deng, S. Refractive index sensor based on twisted tapered plastic optical fibers. Photonics 2019, 6, 40.

[17] Jing, N.; Zheng, J.; Zhao, X.; Teng, C. Refractive index sensing based on a side-polished macrobending plastic optical fiber. IEEE Sens. J. 2015, 15, 2898-2901.

[18] Teng, C.; Jing, N.; Yu, F.; Zheng, J. Investigation of a macro-bending tapered plastic optical fiber for refractive index sensing. IEEE Sens. J. 2016, 16, 75217525.

[19] Liu, G.; Feng, D.; Zhang, M.; Jiang, S.; Ye, Z. Sidehole plastic optical fiber for testing liquid's refractive index. IEEE Sens. J. 2015, 15, 2902-2905.

[20] Feng, D.; Zhang, M.; Liu, G.; Liu, X.; Jia, D. Dshaped plastic optical fiber sensor for testing refractive index. IEEE Sens. J. 2014, 14, 1673-1676.

[21] Teng, C.; Yu, F.; Jing, N.; Ding, Y.; Si, Z.; Zheng, J. Investigation of refractive index sensors based on sidepolished plastic optical fibers. Opt. Fiber Technol. 2017, $36,1-5$.

[22] Liu, L.; Yang, J.; Yang, Z. Theoretical Analysis of the Optical Propagation Characteristics in a Fiber-Optic Surface Plasmon Resonance Sensor. Sensors 2013, 13, 7443-7453.

[23] Polygerinos, P.; Seneviratne, L.D.; Althoefer, K. Modeling of Light Intensity-Modulated Fiber-Optic Displacement Sensors. IEEE Trans. Instrum. Meas. 2011, 60, 1408-1415.

[24] Tang, J.L.; Cheng, S.F.; Hsu, W.T.; Chiang, T.Y.; Chau, L.K. Fiber-optic biochemical sensing with a colloidal gold-modified long period fiber grating. Sens. Actuator B 2006, 119, 105-109.

[25] Sequeira, F.; Duarte, D.; Bilro, L.; Rudnitskaya, A.; Pesavento, M.; Zeni, L.; Cennamo, N. Refractive Index Sensing with D-Shaped Plastic Optical Fibers for Chemical and Biochemical Applications. Sensors 2016, $16,2119$.

[26] Bilro, L.; Alberto, N.; Pinto, J.L.; Nogueira, R. Optical sensors based on plastic fibers. Sensors 2012, 12, 12184-12207.

[27] Danny, C.G.; Raj, M.D.; Sai, V.V.R. Investigating the refractive index sensitivity of u-bent fiber optic sensors using ray optics. J. Lightwave Technol. 2020, 38, 1580-1588. 